\title{
Future Deep Inelastic Scattering with the LHeC
}

\author{
Max Klein (University of Liverpool) \\ Contribution to a Book dedicated to the Memory of Guido Altarelli, January 21, 2018
}

\begin{abstract}
For nearly a decade, Guido Altarelli accompanied the Large Hadron electron Collider project, as invited speaker, referee and member of the International Advisory Committee. This text summarises the status and prospects of the development of the LHeC, with admiration for a one-time scientist and singular leader whom I met first nearly 40 years ago under the sun shining for the "Herceg Novi School" in Kupari, where we both lectured about the beautiful science of Deep Inelastic Scattering and enjoyed life under a yellow moon.
\end{abstract}

\section{Introduction}

The time is now 50 years after the birth of deep inelastic scattering (DIS) with the discovery of partons [1, 2] and 40 years after the paper of Altarelli and Parisi, cited over six thousand times, on "Asymptotic Freedom in Parton Language" [3]. We are 20 years after the approval of the huge LHC detectors, ATLAS and CMS. These have now taken about $100 \mathrm{fb}^{-1}$ of data each. Adding the publications of all LHC collaborations, nearly two thousand papers have appeared, based on the superb performance of the Large Hadron Collider. The Higgs boson was discovered five years ago. About this most impressive harvest Guido Altarelli had noted, we expected complexity but we found a maximum of simplicity [4], commenting not on the hugely complex experiments nor ingenuitive, novel analysis techniques but refering to the surprising absence of new physics, besides the exciting observation of the Higgs boson. No evidence was observed so far in support for extra dimensions, symmetries, particles nor a grand unified theory. Guido thought about this deeply: ..it is true that the SM theory is renormalizable and completely finite and predictive. If you forget the required miraculous fine tuning you are not punished, you find no catastrophe! .. The possibility that the Standard Model holds well beyond the electroweak scale must now be seriously considered.. We are experiencing a very puzzling situation but, to some extent, this is good because big steps forward in fundamental physics have often originated from paradoxes. We highly hope that the continuation of the LHC experiments will bring new light on these problems... [4].

The LHC is now projected to operate for two decades hence, with a major interrupt for accelerator and detector upgrades in between. A new horizon for these explorations would be opened with an additional $\mathrm{ep} / \mathrm{e} A$ experiment, i.e. with electron-proton (and electron-ion) collisions concurrent to the default LHC operation in the thirties. The Large Hadron electron Collider (LHeC) was principally designed in an extended concept report (CDR) [5]. This was published in June 2012, a few weeks before the Higgs boson discovery was announced. It considers the addition to the LHC of a $60 \mathrm{GeV}$ energy electron beam accelerator, arranged tangential to the main ring of $27 \mathrm{~km}$ circumference. This configuration is thought to form a novel $e p$ and $e A$ experiment which would be able to collect $\mathrm{O}(1) \mathrm{ab}^{-1}$ of integrated luminosity, exceeding the HERA result by a factor of thousand and its kinematic range by nearly 20. Its salient feature will be the synchronous operation of $e p$ with $p p$ such that no principal loss of LHC luminosity shall occur. The LHeC would enable electron-hadron energy frontier physics explorations at about $1 \mathrm{TeV}$ cms energy, which is four times higher than future Higgs facilities, such as in China (CepC) or Japan (ILC), may achieve in electron-positron collisions. The question such a programme of deep inelastic electron-hadron scattering at the LHC has to answer consists of how that may lead to new insight into the SM and beyond, on its own and in complementing the $p p$ and heavy ion physics pursued at the Large Hadron Collider. This paper has 
been written in memory of Guido Altarelli who advised the LHeC development in his unforgettable manner as a member of the International Advisory Committee, a referee to the CDR and with many illuminating presentations and discussions. Together with Lev Nikolaevitch Lipatov, whom we lost so early also, the LHeC had two monumental theoretical physicists on its side, their inspiration, far reaching theoretical insight and high demand for quality.

The article presents brief accounts of the achievements of HERA (Sect.2) as well as lessons from the first years of LHC physics (Sect.3). There follows a description of the LHeC accelerator design including an introduction to its planned testfacility [6] and a sketch of its detector concept (Sect. 4). The LHeC would be the fifth major experiment at the LHC and it has five major themes which are illustrated in five subsequent sections:

- Based on the unique hadron beams of the LHC and employing a point-like probe, it would represent the world's cleanest, high resolution microscope for exploring the substructure of and dynamics inside matter (Sect. 5);

- With concurrent ep and $p p$ operation, the LHeC would transform the LHC into a 3-beam, twin-collider, energy frontier accelerator facility. Through ultra-precise strong and electroweak interaction measurements, the ep results would make the HL LHC facility a much more powerful search and measurement laboratory than current expectations based on $p p$ only could possibly entail (Sect. 6);

- The clean DIS final state in neutral (NC) and charged currents (CC) enables a high precision Higgs physics programme with the LHeC if it reaches $\mathrm{O}\left(10^{34}\right) \mathrm{cm}^{-2} \mathrm{~s}^{-1}$ luminosity. The joint $p p / e p$ LHC facility can become a Higgs factory of unprecedented impact. It would combine high, per cent precision for abundant Higgs decay channels in ep with improved precision on rare channels, high $p p$ luminosity and the resolution of QCD uncertainties through ep. This transforms the view on the Higgs physics potential at the LHC (Sect. 7);

- As a new, unique, luminous TeV scale collider, the LHeC has an outstanding opportunity to discover new physics, such as in the exotic Higgs, dark matter, heavy neutrino and QCD areas, which is actively being studied and will be illustrated in Sect. 8;

- The LHeC leads into the region of high parton densities at small Bjorken $x$. It extends the kinematic range in lepton-nucleus scattering by nearly four orders of magnitude. It so is expected to transform nuclear particle physics completely, by establishing a QCD base for Quark Gluon Plasma (QGP) phenomena and resolving the hitherto hidden parton dynamics and structure of nuclei (Sect.9).

Guido Altarelli delivered his first lecture to the LHeC community at the first CERN-ECFA workshop in 2008 [7], in which he posed questions to work on. In June 2015 he delivered the last scientific presentation of his lifetime, actually to the annual workshop on the LHeC, when all of us had no clue about his health situation. There followed a session of the International Advisory Committee for the LHeC, chaired by CERN DG emeritus, Herwig Schopper, in which Guido had asked: The physics case of the LHeC is essentially made, can we proceed and build it? He knew there would not be a straight answer possible then as we were all aware of things still to be done. This article is an attempt to describe the background of this statement and to sketch how indeed we can build a TeV scale ep/eA experiment, for the HL LHC lifetime and beyond into the HE LHC, should our community find the courage and means for supporting it. Some concluding remarks are made in Sect. 10 .

Guido Altarelli often raised the questions about the value and determination of the strong coupling constant $\alpha_{s}$ and also, since his youth [8], why the longitudinal structure function $F_{L}$ was so hard to obtain. Having spent many years of my life to measure both, I have added an Appendix, written from the perspective of HERA and the LHeC, to this otherwise more general overview. 


\section{Lessons from HERA}

It has become almost a habit to present $p p$ as the discovery machines and $e^{+} e^{-}$as the clean machines, and to forget to mention ep. Be it intention or sloppiness, this has been so often repeated that one may get tired in raising the concern that such a view is indeed unjust with respect to the roles of TeVatron, LEP and HERA, or the role of HERA for the LHC physics, and that it will hinder the prosperity of our science if its future is unwarily narrowed. Guido Altarelli knew that a $2 \rightarrow 2$ scattering of $e$ and $p$ could be viewed as $e e, e p$ and $p p$, just as it had three Mandelstam variables $(s, t$ and $u$ ), and all three reactions had their merits and complementary roles because the electron is a point-like lepton and the proton a composite hadron. The principal recognition of the LHeC development is that $p p$ and $e p$ are a symbiotic unit of different entities, which together would reach out much further than alone. A combined $\mathrm{LH}(\mathrm{e}) \mathrm{C}$ facility, for example, would challenge a possible future $e^{+} e^{-}$collider as $e p / p p$ combined with $e^{+} e^{-}$would unravel the Higgs properties and the possible Higgs link to new physics particularly thoroughly. The HERA ep collider could have discovered the Higgs boson had it had a thousand times higher luminosity, which is the luminosity goal now. The LHeC will surpass its physics by an enormous extent, but it rests on HERA's foundations.

The "Hochenergie Ringanlage" (HERA) was the first electron-proton collider ever built. Its proposal was endorsed in 1984. HERA was operated between 1992 and 2007 with colliding electron (also positron) and proton beams, of energy, for most of the time, of $E_{e}=26.7 \mathrm{GeV}$ and $E_{p}=920 \mathrm{GeV}$. The cms energy was $\sqrt{s}=2 \sqrt{E_{e} E_{p}}=319 \mathrm{GeV}$ with which HERA was suited to explore the physics at the Fermi scale, set by the vacuum expectation value of the Higgs field, $v=\left(G_{F} \sqrt{2}\right)^{-1 / 2}=246 \mathrm{GeV}$. The luminosity eventually reached values of up to $4 \cdot 10^{31} \mathrm{~cm}^{-2} \mathrm{~s}^{-1}$, and a total integrated luminosity of $0.5 \mathrm{fb}^{-1}$ was collected by $\mathrm{H} 1$ and as well by ZEUS in 15 years. HERA established the ep scattering energy frontier and served to a large extent as a search laboratory for new physics especially through configurations characteristic for the spacelike ep configuration such as leptoquarks or $R$-parity violating supersymmetry. Albeit certain fluctuations appeared, especially on leptoquark and exotic multi-lepton signatures, one concluded that in the mass range explored by HERA new particles, with certain couplings, would not exist. H1 and ZEUS confirmed the unification of the electromagnetic and weak interactions at 4-momentum transfers, $\sqrt{Q^{2}} \leq v$, through high $Q^{2}$ measurements of photon and $Z$ exchange in $\mathrm{NC}$ and of $W$ exchange in $\mathrm{CC}$ [9]. HERA discovered a rise of the sea quark, $x \Sigma$, and gluon, $x g$, densities in the newly accessed region of small Bjorken $x$, by precisely measuring the structure function $F_{2}\left(x, Q^{2}\right)$ and its derivative $\partial F_{2} / \partial \ln Q^{2}$, respectively. Despite some peculiarities in the QCD analysis of the NC cross section at low $x$ and $Q^{2}\left[9\right.$, the linear $Q^{2}$ evolution DGLAP equations, the monumental predictions of Guido Altarelli and Lev Lipatov with collaborators, were established to hold in the full HERA range which could be seen as indeed a surprise as the $\ln 1 / x$ terms, characteristic to the BFKL evolution, were large at small $x$. Often overlooked, but of crucial relevance for future low $x$ physics, as $x \propto Q^{2} / s$, we also discovered that the gluon distribution would not rise towards low $x$ but, on the contrary, disappear when $Q^{2}$ was approaching the mass of the proton squared region $M_{p}^{2} \sim 1 \mathrm{GeV}^{2}$, at the edge of the DIS kinematic region. Following some initial EMC data on charm production in DIS, HERA also measured the charm and bottom quark densities in the proton and provided an experimental base for their theoretical study in a variety of dynamic schemes of changing flavour numbers which now turn out to be quite relevant for the description of $p p$ data such as on associated $b$ and vector boson production at the LHC. Another striking observation at HERA was the finding that the proton remained intact in about $10 \%$ of the violent $e p$ collisions which gave birth to the field of diffractive DIS. Instrumentation and physics at HERA were largely innovative, and many more important measurements were done [10].

Seen from the LHC, HERA has been the indispensable, only base for adequately describing the parton-parton interactions in $p p$, such as the gluon-gluon fusion to generate the Higgs boson. The field of parton distribution functions, PDFs, was born which prior to HERA was at its infancy [11]. 
For QCD, HERA demonstrated that the structure of matter is much richer than the collinear approximation leading to the classic proton PDFs suggests. Measurements of deeply virtual Compton scattering, diffraction, jets, photons, neutrons opened new areas, supported by impressive theory developments, for generalised, pomeron, unintegrated, photon, pion and neutron structure functions. All of these ought to be studied much deeper for understanding the universe at truly microscopic scales.

HERA kept electron-proton scattering as an integral part of high energy particle physics. It demonstrated the richness of DIS physics and the feasibility of constructing and operating energy frontier ep colliders. It is a testimony of the vision and authority of Bjoern Wiik. What did we learn to take into a next higher energy ep collider design? Perhaps there were three lessons about i) the need for higher energy, for three reasons: to make charged currents a real, precision part of ep physics, for instance for the complete unfolding of the flavour composition of the sea and valence quarks, to produce heavier mass particles (Higgs, top, exotics) with favourable cross sections and, a third reason, to discover or disproof the existence of gluon saturation for which one needs to measure at lower $x \propto Q^{2} / s$ than HERA could; ii) the need for much, much higher luminosity: the first almost ten years of HERA provided just a hundred $\mathrm{pb}^{-1}$. As a consequence, HERA could not accurately access the high $x$ region, and it was inefficient and short of statistics in resolving puzzling event fluctuations; iii) the complexity of the interaction region design when a bent electron beam caused synchrotron radiation while the opposite proton beam generated quite some halo background through beam-gas and beam-wall proton-ion interactions. This we had not seen clearly enough prior to and during the initial phase of the HERA luminosity upgrade.

A key question to the future of Deep Inelastic Scattering, to both its particle and nuclear physics components, is the choice of energy. This is an issue also in the debate [12] about the current EIC designs which have a maximum of luminosity at a $\sqrt{s}$ of either 35 (for the Jlab EIC [13]) or $100 \mathrm{GeV}$ (for eRHIC [14]). This discussion, however, too often leaves the comparison with the LHeC out ${ }^{1}$, which has a $\sqrt{s}$ of $1300 \mathrm{GeV}$. A complete view is desirable, extending first attempts such as [15, as to what can be learned from going back by one or two orders of magnitude in $Q^{2}$ below HERA, as with the US EICs, and what can be achieved by a large increase, as with LHeC or even FCC-eh, and, furthermore, which energy range was crucial to be covered in electron-ion scattering. It would then become clearer as to where real synergy resides. A particular question regards the puzzle on the composition of the proton spin which at HERA was investigated by the HERMES experiment, and has recently been claimed to be resolved in lattice QCD [16]. That does not require maximum energies, as the asymmetries of polarised scattering cross sections to measure vanish proportional to $x$. Very high energy in eh scattering is crucial for a DIS Higgs, top, BSM, PDF, vector meson and low $x$ programme. One crudely may call the low energy EICs extended continuations of the NMC, COMPASS and HERMES research programs, on nuclear structure, QCD and spin, while the LHeC is in the line of $\mathrm{H} 1$ and ZEUS with the important extension to electron-deuteron and electron-ion scattering which HERA was not able to study. A question is whether the global nuclear and particle physics communities have the intention and influence to shape a common future of DIS which HERA has opened and which can only be rich and convincing if the programme as a whole is considered.

\footnotetext{
${ }^{1}$ One here considers, for example, an extension of saturation scales $Q_{s}^{2}$ from 1 to $2 \mathrm{GeV}^{2}$ while it is known that at so low $Q^{2}$ the gluon density at lower $x$ is practically zero, and at the edge of having a meaning anyhow since one is near the limit of application of pQCD. A true discovery of the saturation of a rise of $x g$ requires to be at very low $x$ where densities are large but also in the perturbative region where $\alpha_{s}$ is small, i.e. $Q^{2} \geq 10 \mathrm{GeV}^{2}$. One cannot discover a saturating gluon density in a region where it is non-existing and no nuclear environment amplification, as speculative as it is by itself, can change that conclusion.
} 


\section{Lessons from the LHC}

\subsection{Proton-Proton Scattering}

Expectations for the observation at the LHC of new physics beyond the Standard Model had been almost unlimited as is illustrated, for example, by the comprehensive study of the ATLAS Collaboration on its physics potential which appeared prior to data taking [17. And yet, the first sizeable data sets revealed no new particle nor symmetry. At the EPS Conference 2011 at Grenoble, Guido Altarelli, in a memorable plenary talk, stated it was "too early for desparation but enough for despair". In 2012 ATLAS and CMS discovered the Higgs boson, termed the last and a most crucial component of the SM gauge field theory. As more data had been collected and analysed (at the time of this article LHC delivered a record integrated luminosity of $100 \mathrm{fb}^{-1}$ ) Guido later noted: It is now less unconceivable that no new physics will show up at the LHC... We expected complexity and instead we have found a maximum of simplicity.. The possibility that the Standard model holds well beyond the electroweak scale must now be seriously considered [4].

If today one talks about lessons from the LHC one may emphasise the following observations: i) the LHC and the general purpose detectors ATLAS and CMS, which just celebrate the 25 years since the approval of their letters of intent, and also the other experiments as LHCb, have operated with high efficiency and reliable success owing to the ingenuitive work of thousands of physicists and engineers. The LHC and its experiments comprise the overwhelming majority of all HEP efforts and had they not succeeded, particle physics would be in danger of its existence; ii) there has been no observation of supersymmetric particles of mass up to $\mathrm{O}(1) \mathrm{TeV}$ and no observation of any of the considered extensions of the SM, such as Kaluza Klein excitations of gravitons or new vector bosons $Z^{\prime}$ or $W^{\prime}$, currently up to masses of $\mathrm{O}(5) \mathrm{TeV}$. The absence of physics BSM 2 had been the biggest surprise at the LHC so far; iii) a next lesson at the LHC was the confirmation of the $\mathrm{SM}$ at a striking level of precision, if one considers the measurement of the inclusive Drell-Yan scattering cross sections of the $W$ and $Z$ bosons [18] to about half a percent precision, apart from the also striking $2 \%$ luminosity uncertainty, or the recent measurement of the mass of the $W$ boson to $19 \mathrm{MeV}$ precision [19]; iv) QCD and electroweak theory had been extended to high orders, with the $\mathrm{N}^{3} \mathrm{LO}$ calculation of the Higgs production at the LHC as an outstanding example. The success of the LHC is its challenge. Globally a huge effort is undertaken to upgrade the LHC accelerator and its experiments to highest reasonable, in terms of event pile-up, luminosities. The HL LHC is projected to operate for two more decades hence, and a fundamental question is that about its science base. The addition of $e p$ adds new possibilities for the discovery of physics beyond the SM and it transforms the LHC facility eventually into a precision particle physics laboratory of wider and deeper range than a sole $e^{+} e^{-}$machine can possibly represent.

\subsection{Scattering of Heavy Ions}

The $\mathrm{PbPb}$ runs at the LHC have confirmed the picture coming from RHIC that the medium created in such collisions behaves very early like a very low viscosity fluid whose behaviour can be well described by relativistic viscous hydrodynamics. It is very opaque to particles traversing it, a

\footnotetext{
${ }^{2}$ This has had major consequences especially for the layout of new electron-positron colliders: at CERN and in China circular machine designs reappeared when it was most likely that an electron-positron collider above the scale set by Higgs and top production was an "empty machine". Interesting enough, both the Chinese CepC and the most recent version of the Japanese ILC set $\sqrt{s}=2 E_{e} \simeq v$, in order to measure the Higgs-strahlung from $Z, e^{+} e^{-} \rightarrow Z^{*} \rightarrow Z H$. The coming energy frontier in $e^{+} e^{-}$is nothing but the Fermi scale with a question mark about CLIC which could principally reach $3 \mathrm{TeV}$ at high cost should a new spectrum of particles still be discovered at subsequent LHC runs or/and new theories inevitably led to the conclusion that new physics was in reach at a few $\mathrm{TeV}$ mass.
} 
phenomenon known as jet quenching. The first statement is supported by extensive studies of azimuthal asymmetries far beyond, both in kinematic reach and order of correlations, those that could be studied at RHIC. The second one is provided by a wealth of measurements, including jet yields and shapes both isolated and accompanied by an electroweak boson, which have triggered a huge theoretical activity to understand the modifications of the QCD branching inside a coloured medium, for a review see [20]. Finally, a great surprise came from the measurements of smaller systems, $p p$ and $p P b$, which revealed a not expected smooth transition when increasing the colliding system size for observables (ridge and azimuthal asymmetries, particle species, interferometry,...) that have been traditionally considered as signatures of the creation of deconfined partonic matter close to thermodynamical equilibrium - Quark Gluon Plasma - in high-energy nucleus-nucleus collisions. In short, all hadron-hadron collisions at the LHC were observed to be of collective nature. It therefore needs the electron-ion configuration to i) establish a new level of understanding the parton dynamics inside nuclei in the kinematic range covered by the HI experiments at the LHC and to ii) provide the theoretically sound initial conditions for the macroscopic evolution of the system leading eventually to an understanding of the emergence of that hydrodynamical macroscopic behaviour from the QCD microscopic dynamics.

\section{The LHeC Project}

Owing to the high energy, intense hadron beams of the LHC, CERN has the unique potential for discovery and precision DIS at the energy frontier and it would be a waste not to utilise that as Guido had formulated already ten years ago [7]. The idea of ep collisions with the LHC was considered already at the first LHC workshop in 1984, again at the Aachen workshop in 1990, and ep was presented as a bonus when Chris Llewellyn Smith, then Director General of CERN, announced the approval of the LHC now 25 years ago, for a brief account of ep collider history see the introduction to [5]. The idea was revived when CERN, ECFA and later NuPECC invited a study of how one may realise $e p$ and $e A$ collisions in view of the LHC physics findings and accelerator and detector technology advances. This was the base for the extended conceptual design report (CDR) of the LHeC [5]. It was published in summer 2012. At just that time the Higgs boson was discovered. The CDR had presented a machine of $10^{33} \mathrm{~cm}^{-2} \mathrm{~s}^{-1}$ luminosity, about a hundred times more intense than HERA I. The CDR had recognised [5] that the $H$ boson, predominantly produced in CC DIS, was generated with a cross section of $200 \mathrm{fb}$ in polarised $e p \rightarrow \nu H X$ as large as in $e^{+} e^{-}$. The $H$ production is through radiation from a spacelike $W(\mathrm{CC})$ or $Z(\mathrm{NC})$, two production mechanisms which unlike in $p p$ are uniquely distinguishable. The final state is clean as there is no pileup at the LHeC. This caused a reconsideration of the accelerator design assumptions, especially a modest enlargement of the electron beam current and reduction of the $\beta^{*}$ parameter. Since, moreover, the beam brightness, i.e. the ratio of number of protons per bunch and the proton beam emittance, was found to be almost three times larger than the so-called "ultimate beam parameters", predicted at that time to guard the future LHC and adopted in the LHeC CDR, a path had been found for the LHeC to become a genuine and competitive Higgs physics facility at atobarn reach of integrated luminosity [21, 22]. It is interesting to recall that Guido Altarelli, in all his LHeC presentations, nevertheless emphasised the high precision, large kinematic range character of the genuine DIS programme, see below, which he knew to value so much that Higgs and BSM, as interesting as they could be, were not required by him to justify an $e p / e A$ energy frontier experiment at CERN ${ }^{3}$.

\footnotetext{
${ }^{3}$ This often reminded me on the proposal for what later became the quark discovery ep experiment at SLAC [1, 2] which had stated the main aim would be "to collect data which could be of use for future experiments". One is struck by such reasons when nowadays no less than a discovery of something like Dark Matter is requested for a project to proceed. The two mile SLAC linac was longer than the two times $1 \mathrm{~km}$ linacs of the LHeC, but the physics program
} 


\subsection{Principal Design Considerations}

The LHeC should provide an intense, high energy electron beam to collide with the LHC. The intensity is gauged through the luminosity goal of $\mathrm{O}(1) a b^{-1}$, the energy chosen to achieve a TeV energy collision. At the time of this write up, a cost-physics-energy evaluation takes place with the goal to be able to realise the $\mathrm{LHeC}$ with a most far reaching physics scope. The wall plug power of the default design [5] had been constrained to $100 \mathrm{MW}$. In that configuration two super-conducting linacs, opposite to each other, accelerate the passing electrons by $10 \mathrm{GeV}$ each. This leads to a final electron beam of $60 \mathrm{GeV}$ in a 3-turn racetrack configuration as is illustrated in Fig.1. For measuring at very low $Q^{2}$ and/or determining $F_{L}$, see below, the electron beam energy may be reduced to a minimum of perhaps $20 \mathrm{GeV}$. For maximising the acceptance at large Bjorken $x$, the proton beam energy, $E_{p}$, may be reduced to $1 \mathrm{TeV}$ about. If the ERL may be combined in the further future with a double energy $(\mathrm{HE}) \mathrm{LHC}, E_{p}$ could reach $14 \mathrm{TeV}$. One therefore considers a DIS scattering complex with an energy range between $\sqrt{s} \simeq 300$ to $1800 \mathrm{GeV}$. It thus covers a range from HERA to the TeV region, at hugely increased luminosity and much more sophisticated experimental techniques.

The ERL arrangement, Fig.1, is located inside the LHC ring but outside its tunnel, which minimises any interference with the main hadron beam infrastructure. The electron accelerator may thus be built independently, to a considerable extent, of the status of operation of the proton machine. This is the principal advantage of the Linac-Ring over the Ring-Ring configuration which had been studied to considerable detail in the CDR also.

The chosen energy of $60 \mathrm{GeV}$ leads to a circumference $U$ of the electron racetrack of $8.9 \mathrm{~km}$. This length is a fraction $1 / n$ of the LHC circumference, for $n=3$, as is required for the $e$ and $p$ matching of bunch patterns. It is chosen also in order to limit the energy loss in the last return arc and as a result of a cost optimisation between the fractions of the circumference covered by SRF and by return arcs. That configuration is adopted also as the default for the FCC-he. Tentatively, for the LHC the ERL would be tangential to IP2 which, according to the current plans, is taken by the ALICE detector until the first long shutdown following the three year pause of the LHC operation for upgrading the luminosity performance and detectors. This shutdown is termed LS4 and may begin in 2030. For FCC-he the preferred position is IP L for geological reasons mainly.

The LHeC operation is transparent to the LHC collider experiments owing to the low lepton bunch charge and resulting small beam-beam tune shift experienced by the protons. The LHeC is thus designed to run simultaneously with $p p$ (or $A A$ ) collisions. This tames the cost and optimises the physics return since a concurrent operation with the LHC will have a direct impact on the HL LHC physics programme as is sketched subsequently.

\subsection{Parameters}

Following [23] the parameters of the current LHeC default design may be summarised as follows. The luminosity $L$ of the linac-ring ep collider may be approximated as

$$
L=\frac{N_{p} N_{e} f \gamma_{p}}{4 \pi \epsilon_{p} \beta_{p}} \cdot H_{g e o} H_{b b} H_{f i l l} .
$$

Here, $N_{p}$ is the number of protons per bunch and $\epsilon_{p}$ and $\beta_{p}$ are the proton emittance and betafunctions. The main proton beam parameters $N_{p}$ and $\epsilon_{p}$ are defined by the LHC GPD experiments in concurrent ep and $p p$ operation. The proton beta-function in the electron-proton collision point may be as small as $\beta_{p}=7 \mathrm{~cm}$. Furthermore, $f=1 / \Delta$ denotes the bunch frequency, which for the

requirements are orders of magnitude harder to satisfy. One is tempted to note certain arrogance of ourselves towards nature and its predictability. The present "no BSM physics" situation of the LHC may lead to a reset of our ambitions and expectations towards a more humble understanding of what we may expect and what we should promise. 


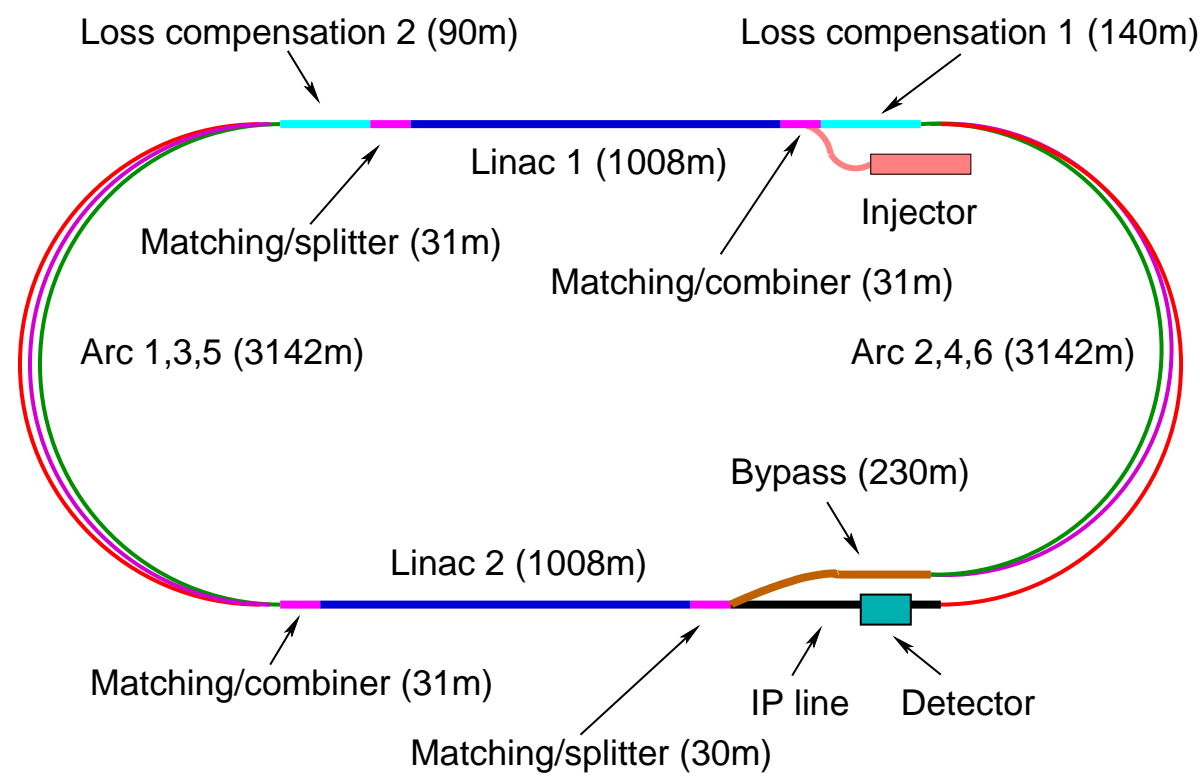

Figure 1: Schematic view of the default LHeC configuration. Each linac accelerates the beam to $10 \mathrm{GeV}$, which leads to a $60 \mathrm{GeV}$ electron energy at the interaction point after three passes through the opposite lying linac structures made of 60 cavity-cryo modules each. The cryomodules comprise four 5-cell cavities at $802 \mathrm{MHz}$ frequency. The arc radius is about $1 \mathrm{~km}$, and the circumference chosen to be $1 / 3$ of that of the LHC. The beam is decelerated for recovering the beam power after having passed the IP. The dump therefore is very ambient friendly.

default bunch spacing of $\Delta=25 \mathrm{~ns}$ is $=40 \mathrm{MHz} . \quad N_{e}$ is the number of electrons per bunch which determines the electron current $I_{e}=e N_{e} f$ with a target value of $15 \mathrm{~mA}$, a twofold increase compared to the conservative CDR. For $60 \mathrm{GeV}$, this will yield a total synchrotron radiation of about $40 \mathrm{MW}$ in the return arcs. To compensate for this power loss through the beam, a grid power of the order of $65 \mathrm{MW}$ may be required. This load may be substantially reduced if the electron energy is diminished.

The factors $H_{g e o}, H_{b b}$ and $H_{f i l l}$ are geometric correction factors with values typically close to unity. $\quad H_{g e o}$ is the reduction of the luminosity due to the hourglass effect, $H_{b b}$ is the increase of the luminosity by the strong attractive beam-beam forces and $H_{\text {fill }}$ is a factor that takes the filling patters of the electron and the proton beam into account. Estimates for these parameters are shown in Tab.4.2. Compared to the CDR of the LHeC from 2012, it seems indeed possible to achieve peak luminosities near to or larger than $10^{34} \mathrm{~cm}^{-2} \mathrm{~s}^{-1}$, which makes these future ep colliders most exciting and efficient machines for the study of new physics at the accelerator energy frontier.

\subsection{The Energy Recovery Development Facility PERLE at Orsay}

When Guido Altarelli had asked, at the 2015 LHeC workshop, whether we could build the LHeC, the IAC, of which he was a very prominent member, discussed the importance of developing the basic technology of the LHeC. Since then a conceptual design report was published [6] of a low energy, energy recovery linac (ERL) facility. A collaboration has been founded with the intent to realise a "Powerful Energy Recovery Linac for Experiments" (PERLE) at the site of LAL Orsay where a hall and parts of the infrastructure already exist. Initially participating institutes are BINP Novosibirsk, CERN, Daresbury, the University of Liverpool, Jefferson Laboratory and the two now merging Orsay Institutes, LAL and IPN. The main parameters of PERLE are derived from the LHeC, such as the electron current of $20 \mathrm{~mA}$ chosen to achieve its $10^{34} \mathrm{~cm}^{-2} \mathrm{~s}^{-1}$ luminosity target.

The PERLE layout considers two accelerating superconducting cavity structures, cryogenic modules each housing four $802 \mathrm{MHz}$ frequency 5-cell cavities, which are embedded at opposite linear 


\begin{tabular}{|l|c|c|c|c|}
\hline parameter [unit] & LHeC CDR & ep at HL-LHC & ep at HE-LHC & FCC-he \\
\hline$E_{p}[\mathrm{TeV}]$ & 7 & 7 & 12.5 & 50 \\
$E_{e}[\mathrm{GeV}]$ & 60 & 60 & 60 & 60 \\
$\sqrt{s}[\mathrm{TeV}]$ & 1.3 & 1.3 & 1.7 & 3.5 \\
bunch spacing [ns] & 25 & 25 & 25 & 25 \\
protons per bunch $\left[10^{11}\right]$ & 1.7 & 2.2 & 2.5 & 1 \\
$\gamma \epsilon_{p}[\mu \mathrm{m}]$ & 3.7 & 2 & 2.5 & 2.2 \\
electrons per bunch $\left[10^{9}\right]$ & 1 & 2.3 & 3.0 & 3.0 \\
electron current $[\mathrm{mA}]$ & 6.4 & 15 & 20 & 20 \\
IP beta function $\beta_{p}^{*}[\mathrm{~cm}]$ & 10 & 7 & 10 & 15 \\
hourglass factor $\mathrm{H}_{g e o}$ & 0.9 & 0.9 & 0.9 & 0.9 \\
pinch factor $\mathrm{H}_{b b}$ & 1.3 & 1.3 & 1.3 & 1.3 \\
proton filling $\mathrm{H}_{f i l l}$ & 0.8 & 0.8 & 0.8 & 0.8 \\
luminosity $\left[10^{33} \mathrm{~cm}^{-2} \mathrm{~s}^{-1}\right]$ & 1 & 8 & 12 & 15 \\
\hline
\end{tabular}

Table 1: Baseline parameters and estimated peak luminosities of future electron-proton collider configurations for the electron ERL when used in concurrent $e p$ and $p p$ operation mode, taken from 23.

parts in a racetrack configuration with two triple arcs. Operation for ERL is in CW mode at typical gradients of $18 \mathrm{MV} / \mathrm{m}$. An electron beam of up to $20 \mathrm{~mA}$ current, derived from a DC photocathode, is injected with $7 \mathrm{MeV}$ energy into the PERLE lattice which is an ellipse of main dimensions $5.5 \times 24 \mathrm{~m}^{2}$. With three turns the beam is accelerated to about $500 \mathrm{MeV}$ and, after a $\pi / 2$ phase shift, decelerated and dumped at the injection energy. PERLE therefore represents a unique facility with its multi-turn ERL and a new, high power level of $10 \mathrm{MW}$ which distinguishes it from various other facilities at lower energy or different current, frequency or return arc magnet technology.

The frequency of $802 \mathrm{MHz}$ is chosen to comply with the LHC constraint following an optimisation involving beam breakup stability for the LHeC, cost of the structure and RF power as well as cryogenic capacity [24, 25. A 5-cell Niobium cavity prototype for PERLE, designed by CERN and Jlab 6], has been built at Jefferson Laboratory in 2017 and successfully passed all checks for mechanical stability, tolerance and flatness. Very recently its $Q_{0}$ factor, which determines the maximum of stored energy relatively to the input power, has been initially measured and was observed to safely surpass the requirement of a value in excess of $10^{10}$, see Fig.2. The development of PERLE is thus passing its first and a crucial milestone, with further investigations as on the field emission to possibly follow and elements for higher order mode extractions under design.

Currently the PERLE Collaboration works on the technical design of the facility with emphasis on building the first cryomodule, using the considerable experience gained with the existing SPL, ESS and SNS modules developed by CERN, IPN Orsay and Jlab, respectively. It is hoped that PERLE can start operation within a few years time, also building on the large DC photocathode experience at Daresbury Lab and on the art of magnet fabrication at BINP Novosibirsk. Primarily, PERLE is built as an accelerator $\mathrm{R}+\mathrm{D}$ facility. Questions such as on the time structure, transverse and longitudinal matching, source limitations, space charge, beam break up, halo and other effects ought to be investigated in order to master ERL technology and dynamics at the $10 \mathrm{MW}$ power level.

The high intensity and chosen energy make PERLE in the mid term future a unique facility for the study of photo-nuclear reactions and also particle and nuclear electron beam physics on, for example, the study of the scale dependence of the weak mixing angle, a precise measurement of the proton radius or the search for dark light photons. Other applications, also described in the PERLE CDR [6], comprise the beam based development of SCRF, the test of accelerator components, such as the quench behaviour of superconducting magnets, or detector developments. With a photon intensity 


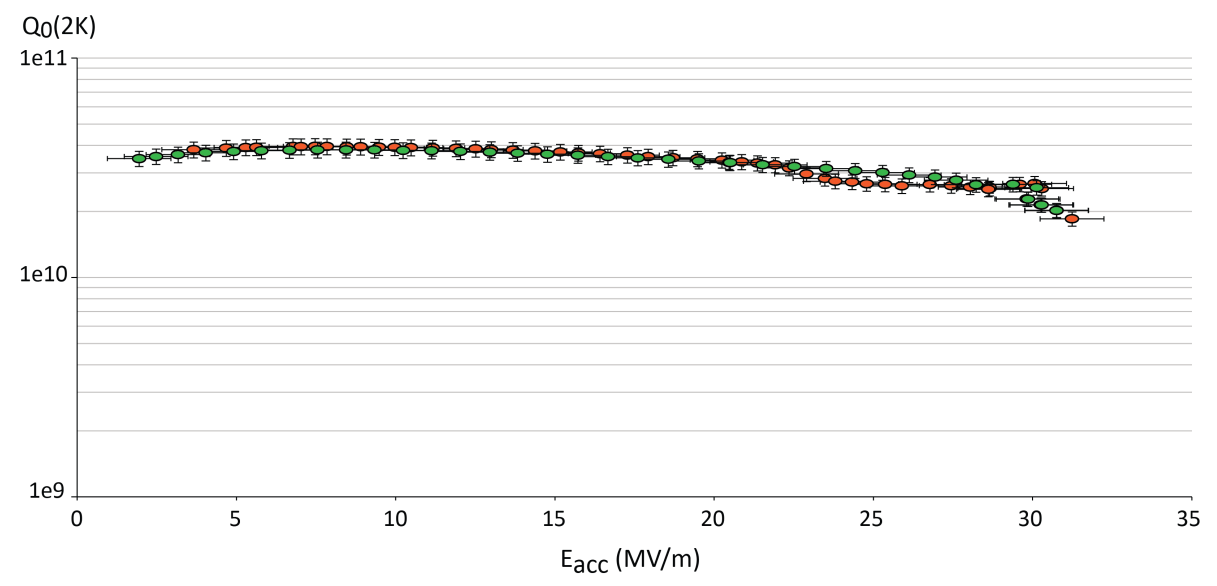

Figure 2: Quality factor $Q_{0}$ of the first 5-cell, $801.58 \mathrm{MHz}$ frequency, Niobium, PERLE prototype cavity determined as a function of the acceleration gradient. The observed behaviour surpasses by far the expectation, with a $Q_{0}$ well exceeding $10^{10}$ and an apparent very weak degradation towards gradients extending much beyond the considered $\mathrm{CW}$ operation working point of $18 \mathrm{MV} / \mathrm{m}$, from [26].

exceeding by orders of magnitude that of the ELI facility [27] currently under construction in Southern Europe, PERLE represents a far reaching new accelerator base for the laboratories in the Orsay and Saclay area, the further collaborating institutes and future possible users for fundamental and applied physics investigations. PERLE may also be configured as a powerful laser physics facility when complemented with an undulator system. At the same time, experience and technology emerging from PERLE are enablers for the LHeC, the highest energy ERL application so far considered.

\subsection{Detector}

The arrangement of the electron ERL tangential to the LHC defines one interaction region (IR) for one detector. By LS4, now scheduled for 2030, the ion-ion program of the LHC ends in order to maximise the $p p$ luminosity collection, and currently the operation of the ALICE detector at IP2 is considered to then be complete. This opens the opportunity for replacing it with a new detector for precision electron-hadron physics. A study has been made [28] of the sequence of installing the new detector in IP2. Based on experience with the pre-mounting of the CMS detector and exploiting the modular structure of the $\mathrm{LHeC}$ detector it was established that a time of less than 2 years shall be sufficient for such an "exchange of detectors". Typical shutdown periods of the LHC range from one to nearly three years, and one may expect that the first long shutdown after the upgrade of machine and detectors may no be short for reasons independent of $e p$. This opens the real opportunity for DIS physics with the LHC in the thirties.

In considering the further future one may expect that the LHC be replaced by its double energy version, the $\mathrm{HE} \mathrm{LHC,} \mathrm{following} \mathrm{the} \mathrm{termination} \mathrm{of} \mathrm{the} \mathrm{LHC} \mathrm{operation} \mathrm{currently} \mathrm{envisaged} \mathrm{to} \mathrm{take}$ place in $\sim 2038$ after accumulating 3 or $4 \mathrm{ab}^{-1}$ of integrated $p p$ luminosity. It thus is conceivable that the $e p$ detector may also be used for operation during the HE LHC phase, from likely the end of the forties to two decades hence. It then seemed not illogical to design an $e p$ detector which could cope with first 7 and later $\simeq 13 \mathrm{TeV}$ proton beams. The current status of its design is sketched in Fig. 3. The detector has a classic collider detector structure and its basic design considerations as well as simulations are summarised in the LHeC CDR [5]. Its characteristic feature is a combined magnet structure which has to be inserted between the electromagnetic and hadronic calorimeters, i.e. a dipole magnet for bending the electron beam into and out of the proton beam axis and a $3 \mathrm{~T}$ solenoid currently combined with that dipole. Two versions exist of warm and LAr electromagnetic 
calorimetry.

Recent work focusses on the adaption to evolving detector technology, such as low material, high resolution Silicon detectors, mainly in connection with the LHC luminosity upgrade. The LHeC conditions are comparatively easier with an $e p$ event pile-up of only 0.1 and an about hundred times lower radiation level compared to the LHC. Strong efforts are made to update the IR design from the CDR to new types of quadrupoles of which high field $N b_{3} S n$ actively shielded quadrupoles are currently most promising [29]. The design of the IR is most challenging and at the end of 2017 still work in progress in collaboration between BNL, CERN and Liverpool. Much care and time are also dedicated to the development of DD4HEP/DDG4 based design, simulation and reconstruction software. The central LHeC detector must be completed by forward proton, neutron and possibly deuteron taggers while the backward region has to be instrumented with near axis photon and electron detectors to measure the ep luminosity from Bethe-Heitler scattering and to tag and normalise the photoproduction background to genuine DIS. These taggers have all been considered in the CDR. It is intended to update the CDR detector design within 2018 and to possibly assemble an LHeC Collaboration which would certainly move these design concept studies, currently also involving service routing and material minimization, much forward.

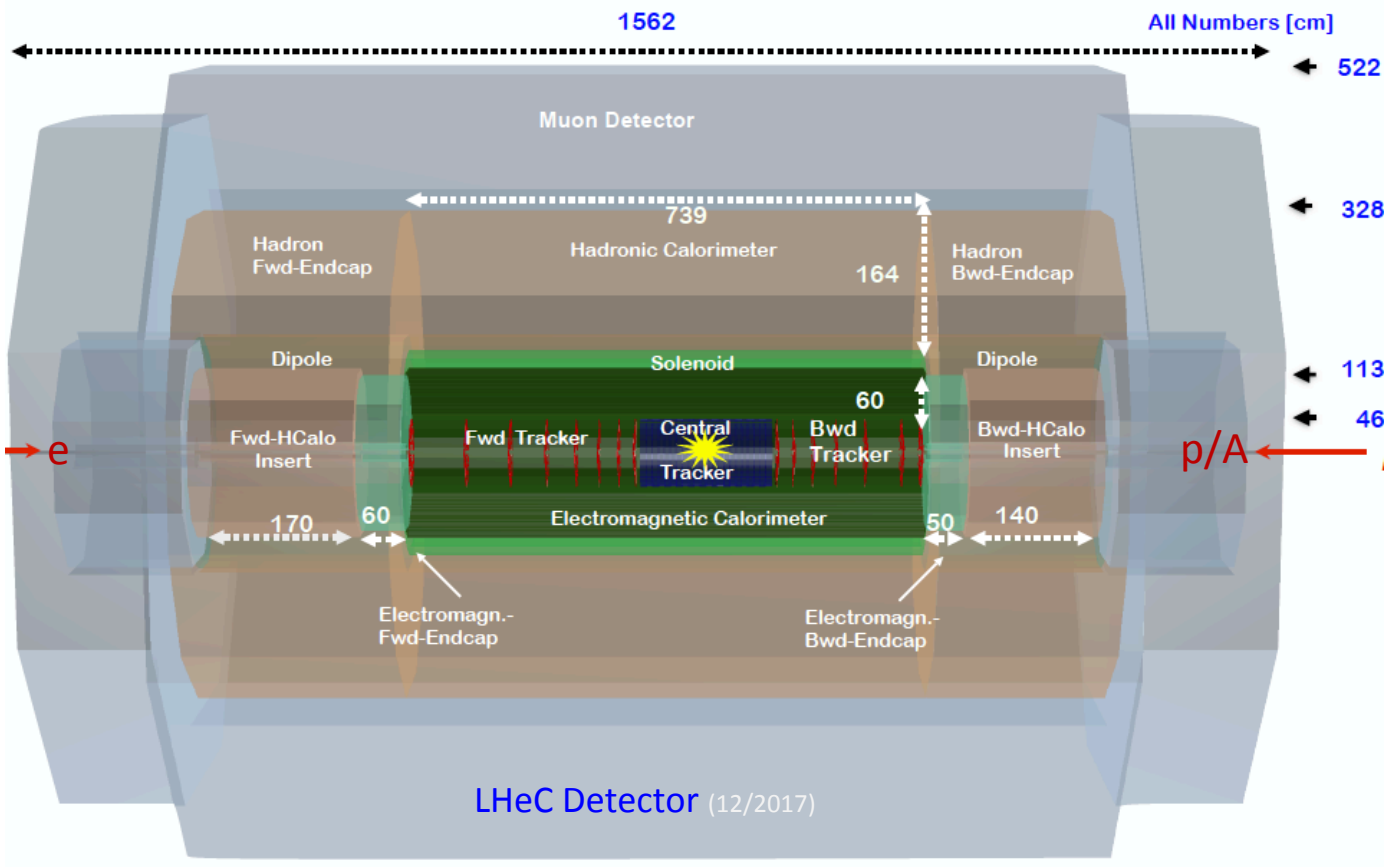

Figure 3: View on the LHeC detector under design to operate with both the high luminosity (HL) and the high energy (HE) LHC. The proton beam comes from the right, pointing to the forward region to which jets and electrons may be scattered of multi-TeV energies. The electron beam points into the backward region which has to measure hadron and electromagnetic energies not exceeding the electron beam energy. The detector is therefore asymmetric. Special attention is required for forward calorimetry and the polar angle acceptance, essential for forward $b$ tagging and for low $Q^{2}$ backward scattered electrons. The beam pipe is eccentric to permit the synchrotron radiation fan of the bent electron beam to pass through the interaction region. The HE (HL) central LHeC detector shown here has a length of $15.6(13.2) \mathrm{m}$ and a radius of $5.2(4.4) \mathrm{m}$. This may be compared with the CMS dimensions of $21 \times 7.5 \mathrm{~m}^{2}$. 


\section{Resolving the Substructure of Matter}

DIS is the means to explore the substructure of matter using photons and $W, Z$ bosons as probes with the kinematics fixed by the scattered electron (NC) or neutrino (CC). Both the (negative) 4-momentum squared, $Q^{2}$, and the parton momentum fraction $x$ are determined externally to the virtual $\gamma(W$ or $Z)$ interaction with the parton. The kinematics can equally well be determined with the hadronic final state. The resolution is $1 / \sqrt{Q^{2}}$, where $1 \mathrm{GeV}$ corresponds to a distance of $0.2 \mathrm{fm}$, which is how Hofstatter discovered a finite proton radius of about $0.8 \mathrm{fm}$ with an electron beam of $200 \mathrm{MeV}$. Following a series of fixed target lepton scattering DIS experiments, with $Q^{2} \leq s=2 M_{p} E_{l}$, HERA made a big step forward by increasing the cms energy squared $s=4 E_{e} E_{p}$ to $10^{5} \mathrm{GeV}^{2}$ in the first $e p$ collider ever built. That range could not be fully explored because of the low luminosity of HERA. The LHeC is designed to achieve a factor of 1000 higher integrated luminosity, and extends the energy squared to $1.7 \cdot 10^{6} \mathrm{GeV}^{2}$. This will lead to quantitatively more valuable and much deeper reaching insight into the structure and dynamics of parton interactions inside matter.

\subsection{The Proton's Partonic Structure and Dynamics}

The momentum distributions of partons inside the proton, $x P\left(x, Q^{2}\right)$, must be determined from experiment. The most suited process to obtain an unbiased determination of the quark and gluon distributions is inclusive deep inelastic NC and CC scattering. "PDFs" as they are now termed have two meanings in that i) they represent a probability view on the substructure of the proton, or similarly of other hadrons, at a given distance $1 / \sqrt{Q^{2}}$, and ii) they describe, supposedly universally through cross section factorisation theorems [30], the hard scattering processes involving partons 4 . Their determination therefore is crucial both for QCD and for searches for new states which makes them so fashionable nowadays at the LHC. DIS is the process to determine PDFs, unlike Drell-Yan scattering, because it is theoretically clean, free of colour interactions in the initial or final state, and it has the prescribable variation of $Q^{2}$ and finally the determination of $x$ external to the intrinsic scattering process.

The PDF programme of the LHeC, as is briefly illustrated below, is of unprecedented depth for the following reasons:

- it will resolve the partonic structure of the proton (and nuclei, see Sect.9.2) for the first time completely, i.e. determine $P=u_{v}, d_{v}, u, d, s, c, b$ and the top and gluon momentum distributions through $\mathrm{NC}$ and $\mathrm{CC}$ cross section and direct heavy quark measurements in a huge kinematic range, from below $x=10^{-6}$ to $x=0.9$ and in $Q^{2}$ from below the DIS region to almost $Q^{2}=4 E_{e} E_{p}=1.7 \cdot 10^{6} \mathrm{GeV}^{2}$;

- a thousand-fold increase of the HERA luminosity, unprecedented precision from new detector technology and the redundant evaluation of the event kinematics from the lepton and hadron final state components will lead to extremely high precision and, technically important, to the fixation of the various PDF analysis parameters from the LHeC data themselves;

- because the high energy CC, unlike at HERA or low energy EICs, becomes equivalent to NC and thus no other data will be required: that is, there is no influence from higher twists or nuclear uncertainties, and no other experiment needed, i.e. LHeC will be the unique base for PDFs, for predictions, discovery and novel tests of theory.

\footnotetext{
${ }^{4}$ In his referee report on the LHeC CDR, in 2012, Guido Altarelli noted on the factorisation theorem in QCD for hadron colliders that: many people still advance doubts. Actually this question could be studied experimentally, in that the $\mathrm{LHeC}$, with its improved precision, could put bounds on the allowed amount of possible factorisation violations (eg by measuring in DIS the gluon at large $x$ and then comparing with jet production at large $p T$ in hadron colliders).
} 
Given the impressive theoretical progress on pQCD, see e.g. [31], one will have these PDFs available at $\mathrm{N}^{3} \mathrm{LO}$ and only then have the PDFs available to respond to the $\mathrm{N}^{3} \mathrm{LO}$ Higgs in $p p$ cross-section calculations demand. For QCD, this will resolve open issues (and probably creating new ones) on $\alpha_{s}$, discussed in the Appendix, answer the question on the persistence (or not) of the linear parton evolution equations at small $x$, see Sect.9.1, and also decisively test whether factorisation holds or not between DIS and Drell-Yan scattering.

The prospect for the determination of the proton-parton distributions with the LHeC has been studied in detail. It relies on the careful simulation of NC and CC measurements, including a full account of their systematic uncertainties and expected correlations, as described in [5, 32]. Two examples are given on the expected precision. Surprising after 50 years of DIS, there is no certainty about the behaviour of the valence-quark distributions at large (and small) $x$. This is illustrated in Fig. 4 which displays the uncertainties and central values for the up and the down valence quarks as a function of $x$ as are obtained in recent PDF analyses. One finds that the up-valence quark is
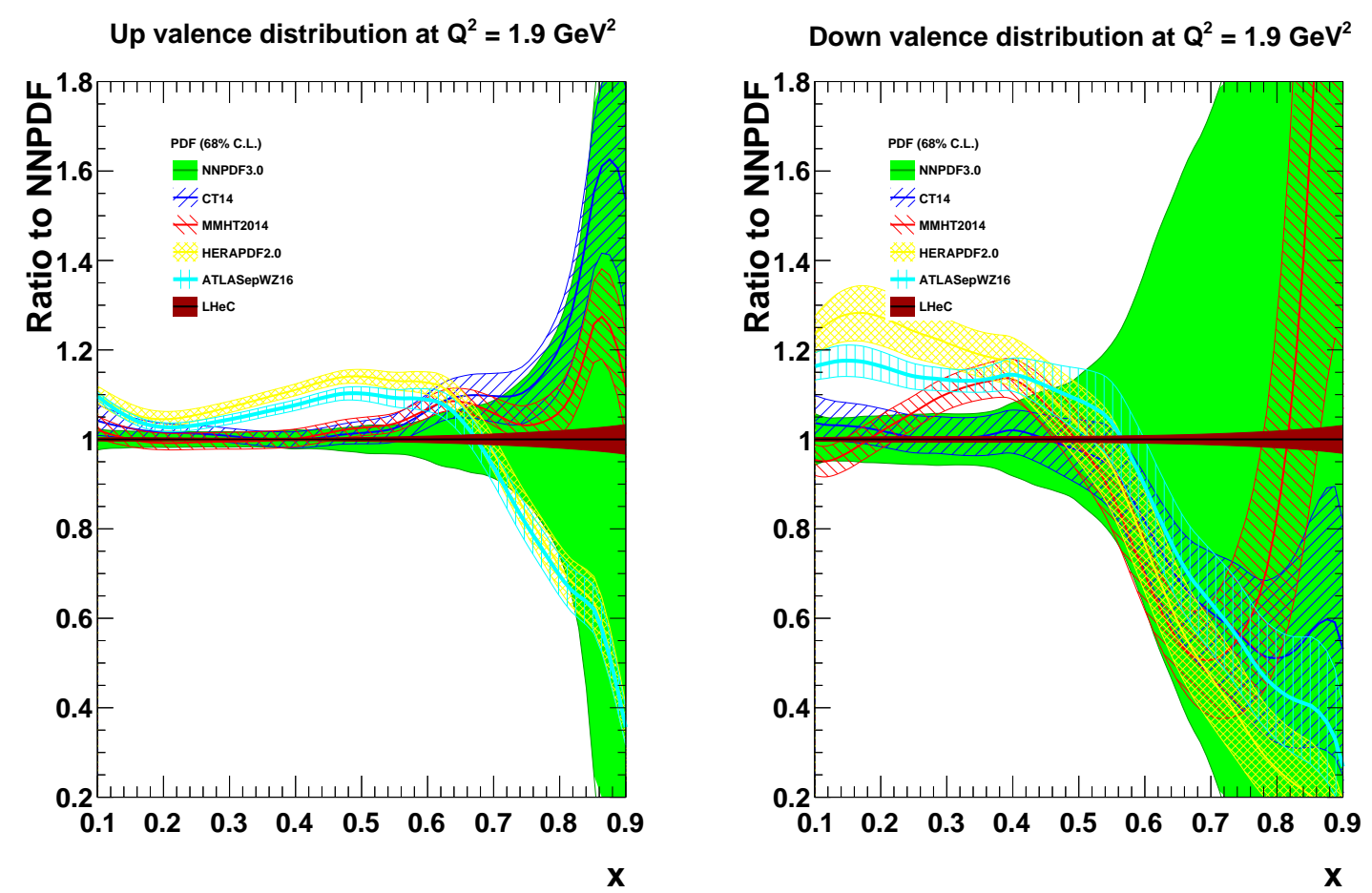

Figure 4: Determination of the valence quark distributions as functions of Bjorken $x$. Plotted are the ratios to the NNPDF result with uncertainties displayed as are provided by the individual sets, left for the up-valence quark and right the down-valence quark distribution. For the LHeC the total uncertainty is plotted and the central value assumed to agree with NNPDF. As non-singlet quantities, the valence quark distributions are approximately the same with varying $Q^{2}$.

better determined than the down-valence quark, which is related to the proton structure function $F_{2} / x$ having the large $x$ limit of $4 u_{v}+d_{v}$. However, at large $x \geq 0.5$ where the distributions decrease, nuclear corrections rise and higher twist effects enter most, we are far from understanding the valence quark distributions. Moreover, there is an apparent difference of $\simeq 20 \%$ between various expectations even at medium $x$. The LHeC will determine both $u_{v}$ and $d_{v}$ with very high precision, see Fig. 4, in the full range of $x$. This is of high interest for QCD, for example concerning the long standing issue of the high $x$ limit of the $d / u$ ratio. For the LHC searches resolving these huge uncertainties is crucial as the luminosity upgraded LHC will explore the high mass Drell Yan regions which currently are masked by the PDF uncertainties, see Sect.6.

One should note that the distributions of the up and down quarks are related, via sum rules, to those of the heavier quarks. This has been demonstrated recently by the ATLAS Collaboration 
which discovered [33] that there should exist a light flavour democracy including the strange quark: finding that the ratio $(s+\bar{s}) /(\bar{u}+\bar{d})$ was about 1 and not suppressed, it was derived 34] that the light sea, $x \Sigma=2 x(\bar{u}+\bar{d}+\bar{s})$ was enhanced by $8 \%$. While ATLAS has derived this result from essentially a moment measurement of $x s$, the LHeC will determine the strange quark distribution from charm tagging in CC directly and as a function of $x$ and $Q^{2}$ over several orders of magnitude as has been demonstrated in the CDR. This is an example of what the difference is between $p p$, the LHC which can still lead to valuable constraints and insight for PDFs, and ep, the LHeC which is the ultimate machine to measure them. Too often that is misunderstood and hopes are expressed one could determine PDFs from $p p$. This is not the case in terms of principles and precision, and it has the philosophical problem, that new physics, hopefully still appearing, as for example a contact interaction effect in $p p$, shall not be misinterpreted as a PDF effect. Two unknowns, PDFs and new physics, require two independent sources of input.
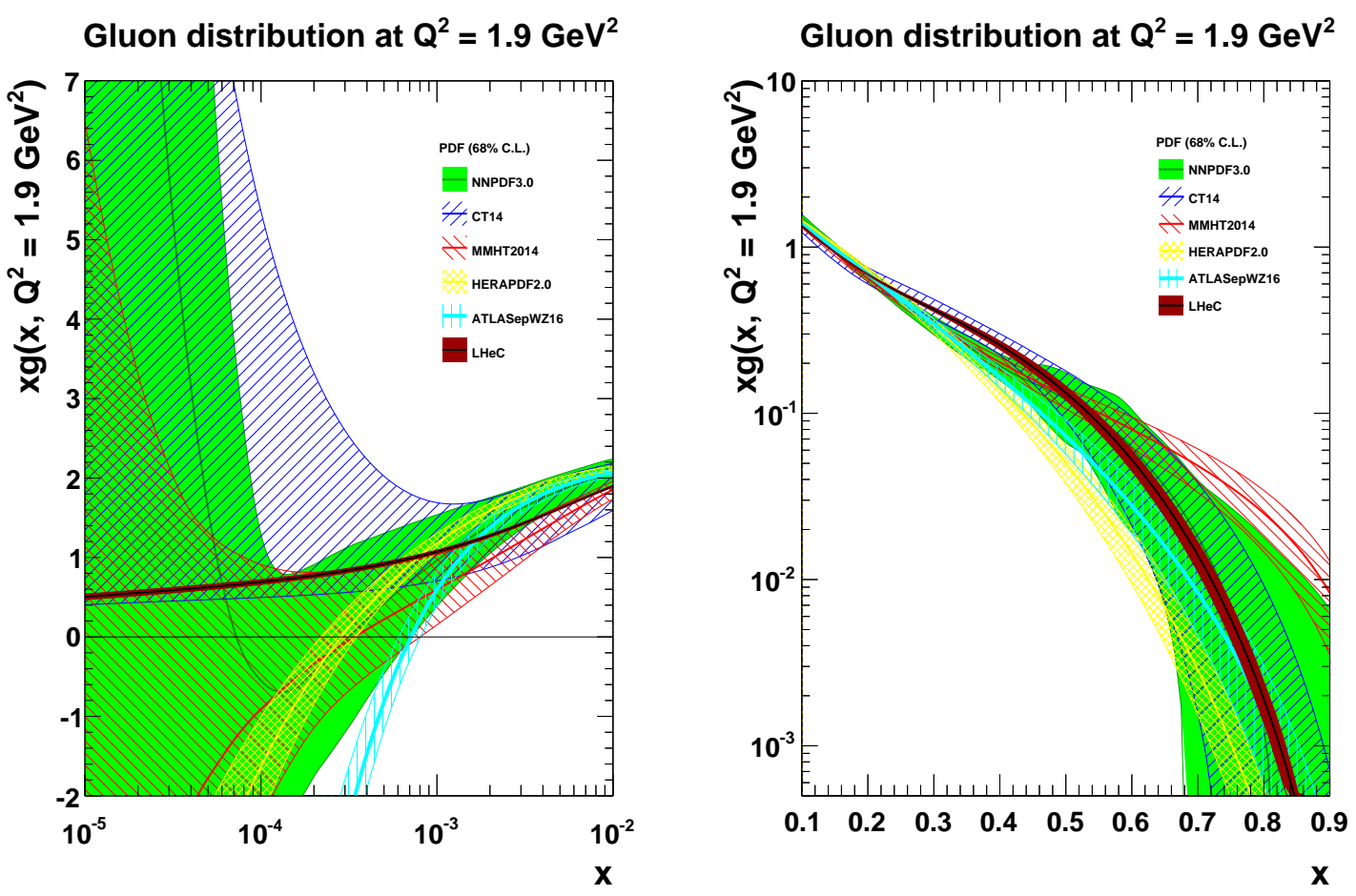

Figure 5: Determination of the gluon momentum distribution in the proton. The expected total experimental uncertainty on $x g$ from the LHeC (dark purple bands) is compared with the most recent global PDF determinations which include the final HERA data, covering for $x g$ a range from $x \simeq 510^{-4}$ to $x \simeq 0.6$, and much of the LHC data from Run I. Left: $x g$ at small $x$; Right at large $x$.

Another important example for the singular PDF prospects with the LHeC regards the gluon distribution. That is of fundamental importance because, as we discovered with HERA, the proton's momentum below $x \simeq 0.01$ is almost completely occupied by gluons, $x g$ is much larger than any for the quark distributions, in the DIS region of $Q^{2}$ exceeding $M_{p}^{2}$ and small $x$. Moreover, the dominant Higgs production channel in $p p$ is gluon-gluon to Higgs fusion, via top loops. The intent to measure the Higgs properties as precisely as possible at the LHC requires to control $x g$ in the region of $x$ between 0.001 and 0.1 to percent precision which is not achieved. The large $x$ behaviour of the gluon distribution is particularly uncertain but especially relevant for testing QCD, as with the factorisation theorem discussed above, and for controlling the production of new states as in gluino pair production, see Sect.6. For reliably measuring $x g\left(x, Q^{2}\right)$ on needs to accurately measure the $Q^{2}$ derivative of the DIS cross section, which, not surprisingly, came out as the only way to accurately access $x g$ at HERA, not diffraction, not $J / \psi$ production, neither open charm which are 
all theoretically less clean and experimentally not competitive with inclusive DIS.

The current status on the determination of $x g$ is illustrated in Fig. 5. A HERA physicist recognises the limits: the determination of $x g$ from $\partial F_{2} / \partial \ln Q^{2}$ at HERA reaches not below $x_{\text {min }} \simeq$ $2 \cdot 10 /(0.7 s)=3 \cdot 10^{-4}$ where the factor of 2 is due to the splitting function, 10 is the minimum $Q^{2}$, 0.7 the maximum $y$ and $s=10^{5} \mathrm{GeV}^{2}$. There arises consequently a huge uncertainty of $x g$ below such $x_{\text {min }}$ as is obvious from Fig. 5 (left). Practically there is a very large uncertainty for $x$ below 0.001. We therefore had not been able to establish the gluon density and neither small $x$ theory subtleties as resummation or BFKL effects at HERA. There are attempts to constrain $x g$ at lower $x$ through heavy flavour production at the LHC. The real determination of the gluon density at small $x$ in the appropriate theory context has to wait for the first LHeC data. The prospect is seen in Fig. 5. There, one also recognizes the HERA limits on the $x g$ determination at large $x$. The gluon density at $x=0.7$ is uncertain by two orders of magnitude, it could be equal to 0.001 but also equal 0.01 or 0.1 . The prospects to derive $x g$ with the LHeC are outstanding, numerically one finds a total (statistical and systematic) uncertainty, at $Q^{2}=1.9\left(M_{Z}^{2}\right)$ of $5.3(0.2), 0.6(0.2)$ and $21(2) \%$ at $x=5 \cdot 10^{-5}, 0.01$ and 0.7 , respectively. The $Q^{2}$ evolution helps in reducing the uncertainty, it yet needs to be verified at the corresponding level for which, at small $x$ one will need the complementary access on $x g$ via $F_{L}$, see the Appendix.

\subsection{DIS and Confinement}

The LHeC nowadays is often reduced to a PDF delivery machine or a Higgs facility. While these may currently indeed be its most striking characteristics, there is a wealth of far reaching, genuine DIS physics ahead with the LHeC. QCD may break, become embedded in a higher symmetry, odderons and instantons await to be discovered. Many fundamental questions are not answered while just been raised as with HERA. That regards, for example, the structure of the deuteron, neutron, pion, nuclei, photon and that of the diffractive exchange often called Pomeron. It comprises the many ways to modify and extend the collinear picture of parton distributions such as the transverse momentum dependent unintegrated parton distributions, the amplitude based generalised parton distributions or the non-DGLAP evolving partons. Jets and jet's substructure, characteristics of quark and gluon jets, vector mesons - a huge field of unexplored physics is awaiting an LHeC experiment. The top quark will appear light when $Q^{2}$ exceeds $M_{t}^{2}$ which holds for the LHeC. The theory of heavy flavour production in $e p$ is not tested well enough, such that fixed flavour or variable flavour number schemes coexist which causes important principal uncertainty in QCD and practical uncertainty for predictions for LHC processes. DIS at high energies is a means to precisely test the electroweak theory in the space-like region with unique measurements as of the scale dependence of the weak mixing angle from below the $Z$ mass up to $1 \mathrm{TeV}$, or of the weak neutral current couplings of light quarks. The LHeC therefore will open a new laboratory for deep inelastic physics of hitherto unseen importance, being well comparable to the present GPD LHC experiments which it is designed to complement. Two examples may illustrate this rich potential.

QCD, as much as it seems to work for the description of high energy interactions as at the LHC, still has a very fundamental problem that is the explanation of the confinement of partons inside the proton. This caused Jaffe and Witten, in 2000, to propose including Quantum Yang-Mills Theory as one of the seven millenium prize questions, in which they indicated that QCD indeed shall be a consistent non-perturbative Quantum Field Theory which has to have three features: i) a mass gap between confined massless gluons and their massive bound states, ii) confinement of partons compatible with $\mathrm{SU}(3)$ invariant free hadrons and iii) chiral symmetry breaking to incorporate current algebra [35]. Thus there is much more behind the strong interaction theory than is naively known. Deep inelastic scattering is ideally suited to approach the confinement puzzle by i) studying the stunning phenomenon of DIS diffraction where the proton remains intact despite a hugely energetic 
collision, and ii) by exploring the process of hadronisation, i.e. the propagation of partons in spacetime, their interaction and the coalescence into colour-singlet states. The role the LHeC can play in these investigations has been discussed in the CDR, see also [36]. The LHeC allows to follow the particle formation, sketched in Fig.6, over a long distance which can be prescribed with the scattering kinematics. Measurements of Deeply Virtual Compton Scattering (DVCS) processes can establish a

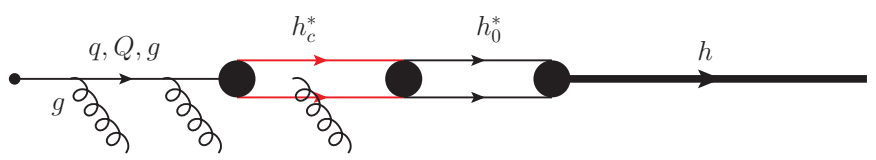

Figure 6: Time evolution of hadronisation from a parton created in an interaction on to the formation of coloured and white states preceding the emission of a hadron, from [36].

dynamic picture of the 3D structure of the proton as is entailed in Generalised Parton Distributions. In this area, the polarised-polarised eh scattering configuration at low energies provided by a future EIC and the high energy LHeC microscopy may indeed well complement each other. Important further insight is gained with the study of the hadronisation process in protons and nuclei, especially with the variation of the energy transfer $\nu=E_{e}-E^{\prime}$.

\section{Empowering Physics at the LHC}

The LHC has currently delivered $100 \mathrm{fb}^{-1}$ of integrated luminosity to ATLAS and CMS. In 2012 both discovered the Higgs boson, with about $10 \mathrm{fb}^{-1}$, but since then, despite many expectations and predictions no further particle or symmetry beyond the SM. LHC physics therefore focusses on two avenues, both requiring a substantial increase of the luminosity: i) searches for new phenomena at the edges of phase space, i.e. states of high mass $M \propto \sqrt{s x_{1} x_{2}}$ initiated by the interaction of two partons carrying momentum fractions $x_{1,2}$ of their parent protons, and ii) measurements of maximum precision to test the SM at a new level. The addition of the LHeC is the ideal complement for both tasks, and it would be crucial also in the interpretation of new physics should that appear. Empowering the physics at the LHC through ep is related to the ultimate precision and clarity with which PDFs and the strong coupling constant will be determined at the LHeC as is discussed in this article.

The physics motivation, as Guido taught us, for the LHeC is tightly related to the future continuation of the hadron collider experiments, HL LHC and FCC [37]. With increasing luminosity the LHC accesses larger and larger masses $M$. A severe problem into which it runs is that the discovery reach is thereby moved into the region of large Bjorken $x$ where the gluon as well as the valence and sea quark distributions are basically unknown. This is illustrated in Fig. 7. The left side shows the uncertainty of a prediction of gluino pair production in the MSSM $g g \rightarrow \tilde{g} \tilde{g}$. At the HL LHC one may reach values up to $4 \mathrm{TeV}$ corresponding to an average $\sqrt{x_{1} x_{2}}$ of about 0.5 , or even higher depending on couplings to new physics. One observes how the uncertainty rises with $M$ which degrades the limit values one may wish to set and complicated an interpretation should there be new physics appearing. The right side shows the uncertainty of the prediction for heavy $W$ bosons as are expected in grand unified theories. The uncertainty, of the quark initiated process, rises very strongly towards large masses. This effectively acts like a barrier for the high luminosity upgrade: huge efforts are made to access high masses, but the proton structure is too uncertain for safely exploring the new and most promising area of phase space. The PDF uncertainty is already now the largest one in such searches which currently set limits of $5.1 \mathrm{TeV}$, obtained for sequential coupling and ATLAS data of $36.1 \mathrm{fb}^{-1}$ [38]. The luminosity upgrade necessitates an electron beam upgrade for exploiting its real 
potential. We often hear the statement that all the relevant information on PDFs can directly be obtained from the $\mathrm{LHC}$ without need of the $\mathrm{LHeC}$, that is not really true, certainly not at the same level of precision [37].
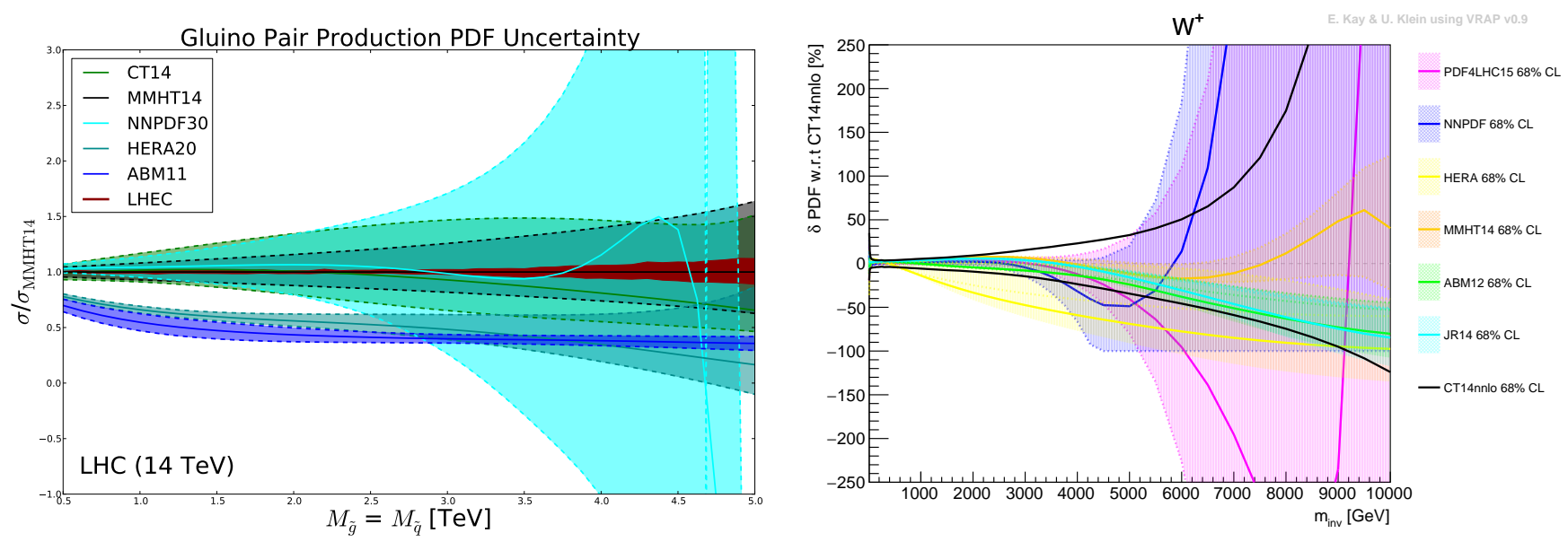

Figure 7: Uncertainties of LHC production cross sections from recent determinations of parton distributions. Left: Gluino pair production as functions of the gluino mass. The inner red band represents the projected LHeC PDF uncertainty [39]. Right: High mass $W^{+}$production [40].

The second avenue of LHC physics, more and more recognised, is "precision", that is the desire and need to test the Standard Model as severely as possible. This concerns primarily the physics of the Higgs boson because its characteristics are not well determined and it may be the portal to new physics, see below. Moreover, the LHC is surprisingly able to perform certain stringent tests of the electroweak theory, with the recent ATLAS measurement of the mass of the $W$ boson [19] to $19 \mathrm{MeV}$ precision as a most striking example. In this result, the PDFs are the single largest systematic uncertainty, of $9.2 \mathrm{MeV}$, resulting from quite a magic cross correlation analysis, which works through the constraint DIS data impose on the total light quark sea, and by considering only a restricted set of PDFs. The LHeC is estimated to predict this $M_{W}$ measurement to $2.8 \mathrm{MeV}$ precision as derived from the uncertainties of the LHeC PDF set [41] which eliminated the PDF problem in the $M_{W}$ mass measurement at the LHC. It is moreover interesting to note, that $M_{W}$ can also be extracted from the LHeC data as a fundamental parameter of the electroweak theory, with an estimated total uncertainty of $[14(\exp )$ and $10(\mathrm{pdf})] \mathrm{MeV}$ [42]. This is an example of the ultra high precision the LHeC will provide in the development of the SM and its possible eventual failure. Many other examples could be discussed here too, such as the scale dependence of the weak mixing angle, the determination of the $V_{c s}$ and $V_{t b}$ CKM matrix elements or the per mille accurate measurement of $\alpha_{s}$ (cf Appendix).

\section{The LHC as a Precision Higgs Physics Facility}

It is crucial for future experiments at the LHC and elsewhere to confirm the properties of the Higgs and the absence of new physics [43]. The Higgs mechanism generates the mass of the weak gauge bosons and the elementary fermions. Its simplest realisation contains a scalar field, $J^{C P}=0^{++}$, which is referred to as the Higgs boson. Following the discovery of this particle, in 2012, much effort has been spent by ATLAS and CMS to pin down its quantum numbers and couplings to its decay products which are expected to depend linearly on their mass squared. The mass of the Higgs boson of $125 \mathrm{GeV}$ implies that a particularly large number of channels is open dominated by the $H \rightarrow b \bar{b}$ decay with a relative probability of $58 \%$ in the SM. Due to the fundamental role of the 
Higgs phenomenon, and in the absence of other new physics, its exploration has moved into the centre of attention at the LHC and for planning the future of collider particle physics.

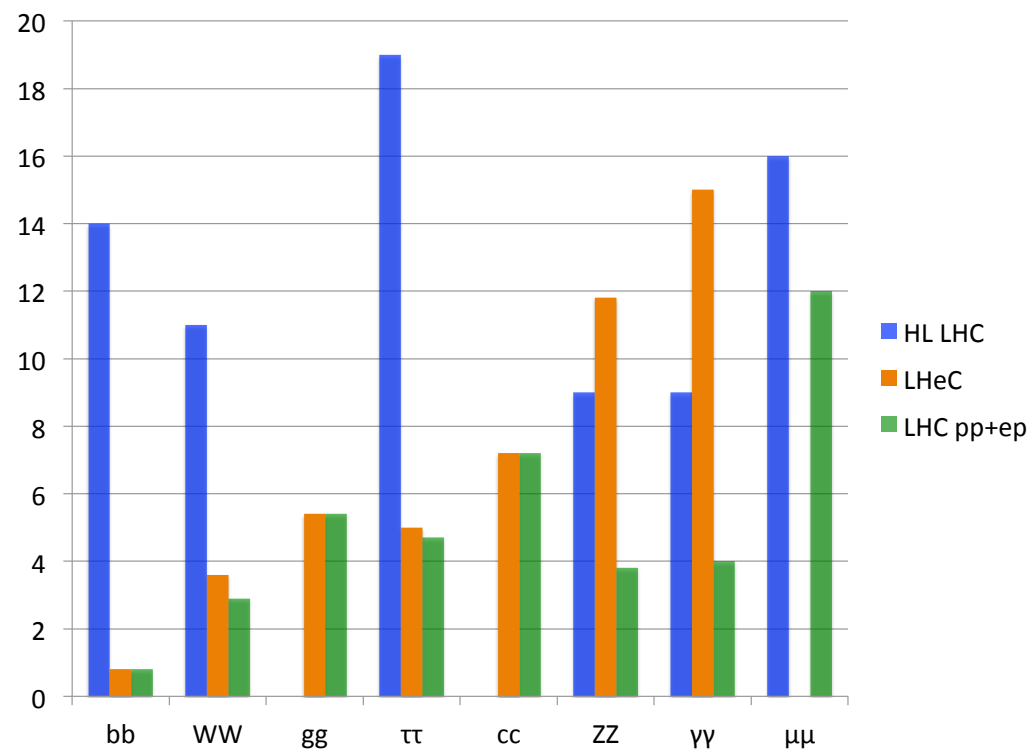

Figure 8: Preliminary estimates of the expected determinations of the relative signal strength $\delta \mu / \mu$, in $\%$, of the Higgs decay to various particles at HL LHC. Blue: the ATLAS expectation for the HL LHC [44; Brown: LHeC prospect 45]; Green: LHC prospect for an $e p / p p$ combination, see text. The decay channels are arranged in descending branching ratio order, from $57.7 \%$ for $H \rightarrow b \bar{b}$ to $0.02 \%$ for $H \rightarrow \mu \mu$. The improvement for all channels wrt. the HL LHC is due to new information on various decays from the $\mathrm{LHeC}$ and to an essential removal of the QCD uncertainties with the provision of $\mathrm{N}^{3} \mathrm{LO}$ precision PDF and $\alpha_{s}$ measurements by the LHeC. An empty column, such as for the LHeC (brown) in the $\mu \mu$ channel, indicates that this may not be accessible to notable precision.

In this context the first question concerns the potential of the HL LHC for probing the Higgs properties. The LHC is the only existing Higgs facility and for very long time it will be the only one with reliable access to very rare decays such as $H \rightarrow \mu \mu$. At the same time it has considerable difficulties to accurately measure certain abundant decays, for which one needs to employ the associate vector boson production, which just recently allowed the $H \rightarrow b \bar{b}$ decay to be recognised [46, 47]. There are, moreover, considerable theoretical uncertainties, partially related to the insufficient control of $x g, \alpha_{s}$ and non-availability of genuine $\mathrm{N}^{3} \mathrm{LO}$ PDFs which can be cured with the LHeC. It has been argued that the LHC can be "turned into a precision Higgs facility" [48] with the addition of the LHeC which has no pile-up and a clean final state signature which distinguishes $W W \rightarrow H$ from $Z Z \rightarrow H$ production. Considerable work is ongoing to study this at the required level, and initial results and the direction of this exploration are illustrated here. In addition, as sketched in Sect.8 below, there are unique opportunities with the LHeC to access new Higgs physics, in the production (as of charged Higgs bosons) and decay (as into dark matter), which would lead beyond the SM.

A particular question is whether the total decay of the Higgs boson is exhausted by its branchings into the known particles, such as pairs of $b, W, g, \tau, c, Z, \gamma$ and $\mu$ and a few mixed combinations such as $Z \gamma$. Fig. 8 illustrates the expected precision of the measurement of the signal strength $\mu$ for the eight most abundant decay modes in the SM. The plot shows the total uncertainty, in blue, as estimated by ATLAS [44 for the HL LHC, i.e. $3 \mathrm{ab}^{-1}$ of integrated luminosity. A second column shows the expected precision on $\mu$ for the LHeC for an assumed luminosity of $1 \mathrm{ab}^{-1}$ collected in electron-proton scattering. The results refer to a charged current interaction where the Higgs boson is emitted from a $W$ exchanged in the $t$ channel. The third, green column illustrates the expected accuracy for the combined $p p$ and $e p$ result at the LHC. It is to be noted for the LHeC that so far 
only the $b \bar{b}$ and $c \bar{c}$ results for the LHeC are due to complete simulations [49] while the other numbers are still of tentative nature 5. For the LHC itself one would expect also improvements related to progress in the analysis techniques and a possible final combination of the two experiment results.

Based on the numbers presented in Fig. 8 one may calculate the sum $B_{H}$ of the Higgs boson branching fractions, $b r_{i}, i=b b, W W, \ldots$, and their total uncertainty. The LHC result, assuming complete independence of the $b r_{i}$ measurements, comes out to be $B_{H}(p p)=0.89 \pm 0.12$. The LHeC, assuming it indeed established the $H \rightarrow g g$ decay, which has a branching fraction of $8.6 \%$, to better than $10 \%$ precision, would reconstruct this sum as $B_{H}(e p)=1.00 \pm 0.02$. The combination of $e p$ and $p p$, with the additional reduction of the QCD uncertainty in $p p$ through information from $e p$, can be expected to accurately determine the total sum as

$$
B_{H}(p p+e p)=\Sigma_{i} b r_{i}=1.00 \pm 0.01
$$

This prospective result means that the LHC can indeed be turned into a a very powerful Higgs facility through the addition of $e p$. This is possible at moderate cost compared to other new facilities currently considered to which $p p / e p$ would be the ideal complement. The precise measurements on $b \bar{b}$ and $c \bar{c}$, the identification of the $g g$ and other decays, still under study, the reduction of QCD uncertainties for the $p p$ results, as well as the experimental and the theoretical cleanliness of the $e p$ Higgs production process [51] are crucial improvements the LHeC entails. The result of Eq.2 would narrow any window for exotic Higgs decays, which currently is wide open (to about $30 \%$ ), to a per cent and therefore represent one of the most crucial new tests of the SM. Failure to reconstruct $B_{H}$ to 1 would, on the contrary, be an ultimate hint to the existence of new physics. Further interpretations of that experimental input on $\delta \mu_{i} / \mu_{i}$ may be made in joint fits of the couplings as are frequently done in prospective Higgs studies, such as in the recent EFT based analysis for the Linear Collider prospects [52]. Extra input in $e p$ is gained from neutral current measurements, especially of the $Z Z \rightarrow H \rightarrow b \bar{b}$ reaction.

It should be seen that a SM independent measurement of the sum of branching ratios $B_{H}$ would require to access the total width $\Gamma$. That is considered to be an important advantage of $e^{+} e^{-}$ colliders. It is intended to study this for the LHeC. The neutral current reaction $e^{-} p \rightarrow e^{-} H X$ can be kinematically fully constrained. The sizeable total NC Higgs cross section corresponds to $25 k$ events which ought to be useful for a yet challenging measurement of $\Gamma$. One should further note that only the LHC (and its possible extensions HE LHC and FCC), neither the LHeC nor a future $e^{+} e^{-}$collider, has the possibility to access rare Higgs decay channels. The total $H$ production cross section at the LHeC, very similarly for the ILC or CepC, is $200 \mathrm{fb}^{-1}$. For a luminosity of $1 \mathrm{ab}^{-1}$ this provides $40 H \rightarrow \mu \mu$ events only. It so appears that the combination of $p p$ and $e p$ at the LHC form a unique, very powerful facility to lead Higgs physics in the thirties to an unprecedented art even if some of the here assumed prospects may alter in the course of the ongoing further study.

\section{New Physics at the LHeC}

Compared to the LHC proton-proton interactions, the LHeC is a cleaner configuration because not only it has no pile-up of events but it has a unique NC or CC final state free of colour connection

\footnotetext{
${ }^{5}$ The signal strength result for the prominent $H \rightarrow b \bar{b}$ decay is $0.8 \%$, or a $0.4 \%$ for the coupling determination. This is a most stunning precision and used to optimise the LHeC detector design, especially for forward $b$ tagging acceptance and optimum resolution of the hadronic calorimeter and joint track-cluster reconstruction. The $b b$ decay was initially studied for $e p$ when the physics of LEP x LHC was explored [50]. Looking back at that analysis one recognises the impressive progress in the experimental and multi-variant analysis techniques and values the $10^{34}$ luminosity prospect particularly high. The $c \bar{c}$ decay is considered to be not accessible at the LHC while the preliminary $e p$ result 49 is $7.2 \%$ which transforms to a $3-4 \%$ precise determination of the Higgs coupling to the charm quark.
} 
effects. Compared to the ILC and CepC, with perhaps initially $\sqrt{s}=0.25 \mathrm{TeV}$ energy, it has an about five times higher centre of mass energy. The ep configuration would fuse electrons and partons to leptoquarks, but it primarily is viewed as a space-like probe, with photons and $Z$ bosons in neutral currents and $W^{ \pm}$in charged currents, of the substructure and dynamics of matter. In these interactions, however, new, heavy particles, such as the Higgs boson, may be produced and new phenomena beyond the Standard Model be revealed. With its luminosity prospect [22, 23] of $\mathrm{O}\left(10^{34}\right) \mathrm{cm}^{-2} \mathrm{~s}^{-1}$ the LHeC has a huge potential for new physics to be discovered in ep. One should note that such a luminosity allows the 15 years of HERA data taking to actually be pursued in just one day. None of the two $e^{+} e^{-}$colliders mentioned, which currently have certain probability of being realised, promises to have significantly higher luminosity. The HL LHC, with $p p$ and ep, and a new $e^{+} e^{-}$machine set to $Z H$ production would be a strong triple for the next phase of energy frontier collider physics, in the third decade of our century. This would substantially strengthen CERN's leading role in particle physics while a healthy competition could rise in Asia, prior to the realisation of a next, more demanding step with raising the energy for $p p$ collisions at CERN.

With increasing intensity and novel approaches, the genuine BSM potential of the LHeC is now being studied, in often comparative analyses, and it is generally observed that the BSM potential in $e p$ is much stronger than thought to be hitherto. A new generation of theorists challenges the conventional believes. It is regretful we cannot discuss this with Guido Altarelli who had put to question whether ep could indeed be a competition with $e^{+} e^{-}$or hadron colliders by calling it, in contrast to those, not a main discovery machine [37]. A few examples are here given subsequently which illustrate new avenues and results towards a new exploration of the BSM physics potential in $e p$ which leads further than the CDR, because of the challenges set with the absence of obvious new phenomena at the LHC and because apparently only slowly the LHeC prospect gets recognised.

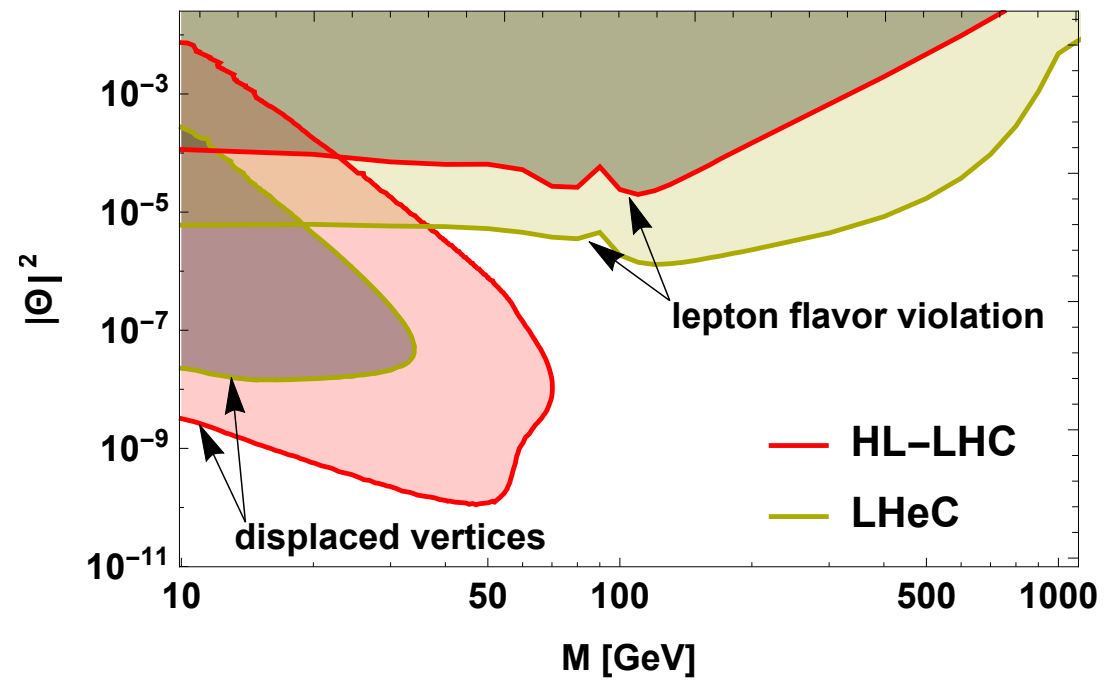

Figure 9: Sensitivity of the HL LHC and the LHeC to the existence of sterile neutrinos in the plane of the active sterile mixing angle $\Theta$ and the heavy neutrino mass $M$. The left, lower mass sensitivity is due to displaced vertex sensitivity while the large mass sensitivity is due to lepton flavour violating multi-jet signatures [53].

A central question in particle physics is that of the origin of mass. The LHeC provides fundamental insight to the origin of baryonic mass, with its far reaching exploration of gluon dynamics in hadrons, of dark mass, through Higgs-to-invisible measurement prospects, of boson and top mass, through unique contributions to their determination and to the Higgs mass, through the approach to the Higgs self-coupling [54]. A recent study [53] draws the attention to the origin of neutrino masses which may require an extension of the Higgs sector or the existence of neutral fermions. Such heavy neutrino mass eigenstates, often termed sterile neutrinos, through their interaction with weak bosons, give rise 
to a rich phenomenology for their possible different production and decay modes, the dependence on mixing parameters or the occurrence of lepton number or lepton flavour violation effects to which the three collider configurations exhibit different sensitivity. Fig.9 displays the result of a comparative study, here focussed on HL LHC and LHeC, of the sensitivity of future collider configurations to the existence of heavy neutrinos, which in $e p$ are primarily produced in the CC reaction $e p \rightarrow N X$. The LHeC reaches a sensitivity of neutrino mass up to $\sim 1 \mathrm{TeV}$ at mixing angles squared down to about $10^{-3}$ extending the $\Theta^{2}$ range of the HL LHC by an order of magnitude to smaller values, owing to the cleanliness of the final state. For lighter masses, up to about $30 \mathrm{GeV}$, very small mixing angles of $\Theta^{2}$ down to $2 \cdot 10^{-8}$ are reached. In this area, from an initial study, the HL LHC sensitivity looks stronger than that in ep, see Fig.9.

Another example for the unique potential to discover new physics at the LHeC has just been presented with a study of the search for long lived Higgsinos [55]. These occur in the MSSM together with Wino and Bino states. Their mixing generates 4 neutralino and 2 chargino mass eigenstates. It has been pointed out that while such particles may be detected at hadron colliders for large mixing, a particularly clean final state is required to discover Higgsinos for small mixing which leads to mass degeneration. The result of quite a thorough analysis of a t-channel $V V \rightarrow \chi \chi$ pair production of supersymmetric particles $\chi$ from exchanged vector bosons $V$ is reproduced in Fig. 10 which displays the number of observed events with at least one long lived particle (LLP) as a function of the chargino mass and lifetime. It can be seen that the LHeC is much more sensitive at small $c \tau$ than the LHC. This advantage holds similarly for exotic Higgs decays which can occur with sizeable branching fractions as is argued in [55] too.

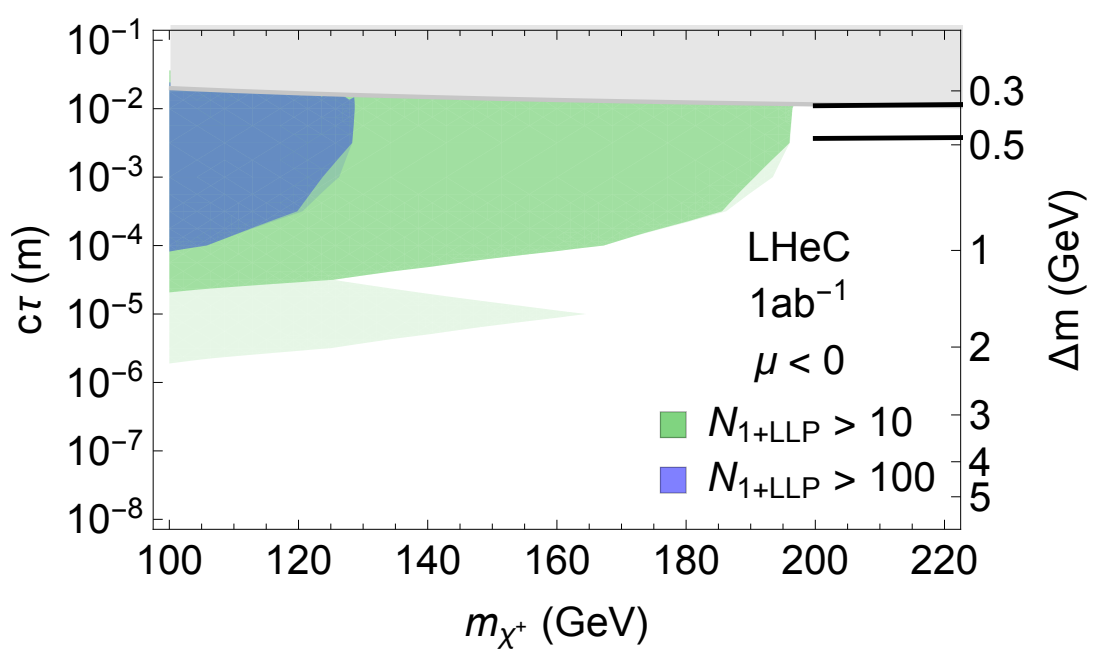

Figure 10: Regions in the mass-lifetime Higgsino parameter plane accessible for LHeC events with at least one LLP. Black curves are projected bounds from searches at the HL LHC, from [55].

A number of further analyses have been published or are being pursued, as are summarised in [49], which demonstrate a very high and often unique potential opened by the LHeC for the discovery of exotic Higgs physics. These regard, for example, the exotic Higgs decays, as into dark matter with a branching sensitivity to $5 \%$, or into two new scalars [56] which provides sensitivities which surpass the HL LHC very much. There are also LHeC related papers published on the top-Higgs coupling [57] on the possible discovery of charged Higgs bosons [58] and others. These and further ongoing studies lead the examination of BSM ep physics much beyond the level reached with the CDR on the LHeC in 2012. They strongly support the conclusion reached in [55] that adding a high luminosity, high energy DIS program to a $p p$ collider "is necessary to fully exploit its discovery potential for new physics" [55]. 
The potential to discover new physics at the LHeC reaches far beyond the Higgs sector and its exotic extension. From very high precision measurements of the structure functions at large $x$ new sensitivity is reached to contact interactions into the $\mathrm{O}(100) \mathrm{TeV}$ region, subject to assumptions on their coupling structure. An analysis of the potential to exploit the general Lagrangian structure as embedded in EFTs has not been done yet for ep even though 1053 out of 2499 parameters characterising deviations from the SM are related to lepton-quark interactions [59].

While HERA had too low energies to study top quark physics, the LHeC is actually a top quark factory with single $t$ and $t \bar{t}$ production cross sections of $\sim 5$ and $\sim 0.02 \mathrm{pb}$, respectively. This permits high precision measurements and unique searches for new physics in many areas, such as the anomalous $t-q-\gamma, t-g-Z$ and triple gauge [60] couplings, the top contribution to the proton's momentum balance (top PDF), anomalous top-Higgs couplings [61] and CP nature [57] and others [62, 63]. The prospects for top ep physics are a particularly strongly developing field with many interesting results supporting the physics case of the LHeC.

New physics can certainly be expected to be discovered in the strong interaction sector. QCD is a very rich and largely unexplored theory regarding, besides the confinement puzzle cited above, for example i) the AdS/CFT duality, which links supersymmetric modifications of QCD to string theory in anti-de-Sitter space or ii) the instanton fluctuations of the gluon field. The LHeC may also discover odderons, a saturation of the gluon density at small $x$, or a breaking of factorisation, as has been discussed in detail in [64]. It should clarify the question why all hadron-hadron interactions of protons and ions, $A A^{\prime}, p A$ and $p p$, exhibit collective phenomena in the expected contrast to ep. The LHeC will push the evolution of QCD much forward, such as the higher order $\left(\mathrm{N}^{3} \mathrm{LO}\right.$ and $\mathrm{N}^{4} \mathrm{LO}$ ) calculations in pQCD, or the physics of non-conventional PDFs. In a list of possible QCD discoveries, presented by C. Quigg with "the unreasonable effectiveness of the SM" at the DIS workshop in 2013 [65], the existence of free quarks, unconfined colour, quark substructure and the embedding of QCD in a higher symmetric theory are included, the discovery of each/any of which would be of far reaching consequences for particle physics. There are strong, accumulating reasons to believe that the LHeC, as the second highest energy, high luminosity, asymmetric collider should indeed be among the main configurations for leading beyond the Standard Model.

\section{$9 \mathrm{LHeC}$ as an EIC}

The prime task of an electron-ion collider at high energies, besides a detailed mapping of nuclear dynamics and structure, is to investigate the QCD dynamics at high parton densities. These are observed at small Bjorken $x$ and may be amplified in a nuclear medium.

\subsection{Low x Physics}

.. subleading terms cannot be ignored and must also be resummed. Their effect is to push the onset of the truly asymptotic regime down to smaller values of $x$, while in an intermediate region, relevant for HERA data, the evolution shows a shallow dip, somewhat lower than the NLO perturbative result. The resummation procedure is determined by solid guiding principles from duality, momentum conservation, symmetry under gluon exchange of the BFKL kernel and accurate implementation of running coupling effects [66]. Deep inelastic scattering is the interaction of leptons and hadrons at four-momentum squared values exceeding the mass of the proton squared, $Q^{2}>M_{p}^{2}$. In this region perturbative QCD may be applied as $\alpha_{s}\left(Q^{2}\right) \simeq 1 /\left(\beta_{0} \ln \left(Q^{2} / \Lambda^{2}\right)\right)<1$, where $\beta_{0}=\left(33-2 N_{f}\right) / 12 \pi$ is the one-loop renormalisation group equation coefficient, $N_{f}$ the number of active flavours and $\Lambda \simeq 200 \mathrm{MeV}$ the famous parameter which quantifies the value of the strong coupling, which is about 0.5 at $Q^{2}=1 \mathrm{GeV}^{2}$. Bjorken $x$ is related to $Q^{2}$ via $x=Q^{2} /$ sy, where the inelasticity $y$ is related 
to the energy transfer $\left(E_{e}-E^{\prime}\right) / E_{e}$ and limited between 0 and 1 . The smallest value of $x$ any ep collider may reach in the DIS region therefore is $x_{m i n}=1 / s$ with $s=4 E_{e} E_{p}$ in $\mathrm{GeV}^{2}$. There is a sequence of minimum $x$ values of $\log x_{\min } \simeq(-2,-3,-4,-5,-6,-7)$ for fixed target DIS, the two US EIC versions, HERA, the LHeC and FCC-eh, respectively. One would call low $x$ the region below $x \simeq 10^{-4}$ down to which the DGLAP equations have been observed at HERA to approximately hold. Low $x$ physics thus is a prime area of research for the LHeC, and for future higher energy DIS colliders under study for CERN and China.

A striking discovery at HERA was the rise of the quark and gluon distributions towards small $x$. It has been argued that unitarity should tame that rise through non-linear parton-parton interactions [67], predominantly gluons because at small $x$ the gluon density is much larger than that of sea quarks. This observation holds only for large $Q^{2}$, in excess of $\simeq 10 \mathrm{GeV}^{2}$, because $x g$ turns valencelike at $Q^{2} \sim 1 \mathrm{GeV}^{2}{ }^{6}$. The key theme of the physics at small $x$ is therefore the understanding of parton interaction dynamics at high densities and for small $\alpha_{s}$, which is an unexplored dynamic state of matter. The key experimental challenge is the discovery of non-linear effects, often synonymously denoted as BFKL dynamics, which would end the region of validity of the linear DGLAP evolution equations. This is not only a question of theoretical importance but of very practical relevance for the LHC and higher energy hadron colliders: the gluon cannot be directly observed, its distribution is defined in a theoretical framework. If that changes fundamentally, the PDFs change and the cross-section calculations alter. Note that in Drell Yan scattering of two partons a state of mass $M$ is produced with a rapidity $\eta$ related as $\sqrt{x_{1} x_{2} s}=M \exp ( \pm \eta)$. For Higgs production the central (very forward) rapidity value corresponds to an average $x=\sqrt{x_{1} x_{2}}$ of 0.009 (0.0002). These $x$ values are lower by a factor of 7 for the FCC. The hadron colliders already sample very small $x$ values for the Higgs but even more so for lighter states. The clarification of the low $x$ dynamics with the LHeC is therefore crucial for the HL LHC and a necessity for the higher energy colliders. It is also of much relevance for the understanding of ultra high energy neutrino physics. These tasks need DIS energies higher than HERA's and not lower.

The decisive test for BFKL dynamics in DIS proceeds through high precision measurements of the salient structure functions $F_{2}$ and $F_{L}$ as has been demonstrated in the LHeC CDR [5]. This holds because these functions are most cleanly theoretically defined. Both, however, are needed because if one had only $F_{2}$ one could attribute changes in the evolution law too easily to parametric peculiarities of the collinear PDFs. This causes a huge challenge for a precision measurement of $F_{L}$ at small $x$ which is discussed in the Appendix.

The establishment of BFKL dynamics at small $x$ would be convincing only if the whole dynamics in this range was consistently described. Consequently there are many other processes under study for the observation of non-linear parton interactions. One example, advocated at a recent LHeC workshop by Mark Strikman, is the production of a vector meson with a rapidity gap in virtual photon-hadron scattering [68]. This reaction is viewed as small dipole elastic scattering at large $t$ and large energies, small $x$, and may lead to an observation of BFKL dynamics. Pursued also in electron-ion scattering it will permit to study the effect of nuclear media with regard to the regulation of shadowing and the eventually possible observation of the black disc regime.

As an experimentalist one admires the considerable theoretical development of low $x$ QCD, indicated also by Guido's remarks cited above, and must admit that the information we have been providing is not enough, HERA being too low in energy and too weak in precision and the LHC being plagued by its nature, the colour connections in the $h h$ interaction. The LHeC, with much less luminosity than $1 \mathrm{ab}^{-1}$, will change that completely and has one of its major and unique tasks in the clarification of the parton dynamics at small $x$.

\footnotetext{
6 That observation is key because it enlarges the effective $x_{\text {min }}$ values by an order of magnitude such that LHeC will investigate the domain down to $x \simeq 10^{-5}$ and eRHIC to only $\simeq 10^{-3}$ which has already been explored with high precision at HERA.
} 


\subsection{Nuclear Parton Dynamics and Densities}

Electron-ion collisions provide rich perspectives for nuclear Chromodynamics. This holds for the low energy EIC collider [69] which may reach cms energies of $\leq 100 \mathrm{GeV}$ as compared to $\simeq 20 \mathrm{GeV}$ by the CERN muon-hadron scattering experiment NMC. It holds as well for the LHeC reaching the TeV energy scale. A difference, apart from the kinematic reach, is the dedication the EIC may put to electron-ion collisions which at CERN would most likely remain in the shadow of the $p p$ program. However, recent evaluations of the $e P b$ luminosity at the LHeC, presented in [23], result in an estimate of $L=710^{32} \mathrm{~cm}^{-2} \mathrm{~s}^{-1}$. One can thus collect annually about $1 \mathrm{fb}^{-1}$ of integrated luminosity which at low $Q^{2}$ is ample, also for semi-inclusive analyses, and implies that a complete DIS programme in electron-ion scattering is ahead including, for example, high $Q^{2}$ charged current physics and providing $\mathrm{O}\left(10^{3}\right)$ Higgs events emerging from electron-lead scattering.

A recent summary of novel perspectives for nuclear physics includes topics "such as the hidden color of nuclear form factors, the relation of the nuclear force at short distances to quark interchange interactions, the effects of color transparency on the baryon-to-meson anomaly in hard heavy-ion colisions, novel exotic multiquark states, the anomalous nuclear dependence of quarkonium hadroproduction, flavour-dependent antishadowing, and the breakdown of sum rules for nuclear structure functions. I also briefly discuss the insights into hadron physics and color confinement that one obtains from light-front holography, including supersymmetric features of the hadron spectrum" [70], too detailed to be discussed in this contribution. It may suffice to state gratefully that Stan Brodsky has had quite an influence on shaping the LHeC programme and supporting its built as a member of the IAC and in his outstanding capacity as a world leading theorist.

For the search for non-linear parton interactions one often cites the expectation, especially when gluon saturation is discussed in the US EIC context, that the gluon density in a nucleus $A$ is amplified by a factor $A^{1 / 3}$, which may only work if the underlying proton parton density is large, i.e. for $x g$ for $Q^{2} \geq 10 \mathrm{GeV}^{2}$ as mentioned above. Moreover, that amplification may be tamed by flavour dependend shadowing effects. There are, principally, two tasks which require independent input: the exploration of BFKL effects and the clarification of nuclear medium and binding effects. It therefore is strongly believed that one needs higher energies than HERA's in order to probe for non-linear evolution in $e p$ and then to compare the result with $e A$. The high luminosity in $e A$ at the LHeC and the large kinematic coverage enable the derivation of the flavour decomposed light sea quarks, the gluon and direct measurements of the strange, charm and beauty distribution in nuclei available at the LHC. This programme is being investigated, for a summary see [71]. It will completely change the way nuclear PDFs are obtained, end the use of $\pi_{0}$ data, for example, and deliver nPDFs free of any pPDF base. One then measures directly the ratio $R=f_{A} / A \cdot f_{p}$, flavour by flavour and for $x$ between a few times $10^{-6}$ up to 1 . This is in contrast to the present method in which $R$ is evaluated using certain set of proton PDFs and a theoretical parameterisation of nuclear effects, with reliable data available only for $x>0.01$. This is a most exciting prospect as has been outlined in [72, 73].

The study of $e A$ collisions in the $\mathrm{TeV}$ regime at the $\mathrm{LHeC}$ would be crucial for the development of nuclear QCD and also for the interpretation of the data coming from the heavy-ion programme. Its importance would be threefold [74]: First, it would greatly reduce our lack of knowledge on nuclear parton densities, that for some observables causes uncertainties of the same size as the eventual signal of high temperature, deconfinement effects. Second, it would establish the QCD dynamics of relevance for the LHC kinematic domain, were it the traditional fixed-order perturbative one or a new non-linear perturbative regime. Finally, it would provide theoretically based initial conditions for the macroscopic evolution of the system and contribute to understanding the emergence of the hydrodynamical macroscopic behaviour from the QCD microscopic dynamics. 


\section{Concluding Remarks}

The future of deep inelastic scattering at high energies can be bright. The opportunity for founding and opening an new laboratory for energy frontier deep inelastic scattering which the LHC provides is indeed unique. It should not be disregarded nor the huge LHC investment be thrown away. The LHeC is a one in a few decades opportunity. Its science programme is unique and rich in its five components sketched here which all are dynamically advancing as a new generation of theorists appears and the LHC physics becomes clearer. The huge step in energy and luminosity, which the LHeC represents, will almost certainly entail surprises, unknown unknowns. It is as well an important step to the development and exploitation of modern, low power, superconducting accelerator technology. The detector represents a challenge many may wish to take up as the design of the HL LHC detector upgrades passes. The LHeC is far more than a mere step for the future of DIS because ep and $e A$ scattering at $\mathrm{TeV}$ energies are recognised to be intimate parts of modern particle and nuclear physics in general. This is underpinned by the observation and design that the $e p$ and $p p$ collisions at the LHC may be recorded synchronously.

The next generation of machines at the energy frontier should comprise the LHeC for it is the only realistic option for energy frontier eh in the coming decades. Not only that the structure of matter is explored deeper, also new particles may be discovered and new dynamics be found. The key carriers of hope and study of modern collider physics, the $W, Z$ bosons, the top quark and the Higgs ought to and can be investigated to sub-percent precision in ep too. A future big step in $p p$ collider energy requires a big step deeper into the dynamics inside the proton.

Particle physics in order to proceed must utilise its rare opportunities, the LHeC is a particularly attractive one, also for CERN's future. It can be realised prior to the next, higher energy $p p$ collider, most likely the HE LHC, which requires more time, more technology developments and substantially larger funds than the LHeC needs. As Guido had put it the physics case of the LHeC is made, can we proceed to build it [75]. So sadly, this was the last word which many of us heard him saying, in his unforgettable style. It is time, 50 years after the discovery of quarks at Stanford, to open a new chapter for high energy electron-hadron scattering for which the LHC is the single and luminous base. The linac to be built is shorter than the one Panofsky and colleagues mastered to build at SLAC.

Acknowledgement The development of the LHeC proceeds because it has been supported by so many exceptional young and elder colleagues whom I just cannot mention here. I want to thank Oliver Brüning for a decade of pleasant and fruitful co-coordination, the CERN directorates under Rolf Heuer and Fabiola Gianotti for their supportive attitude, the various active convenors on LHeC subjects, the FCC coordinators Michael Benedikt and Frank Zimmermann, and the members of the International Advisory Committee led by Herwig Schopper for their encouraging and critical evaluation of the progress. The recent move towards the PERLE facility at Orsay would not have been possible without the conviction of Achille Stocchi, shared with many of us and not least the admirable teams of accelerator scientists at CERN, Jlab and other institutes. For this article I enjoyed special help by Claire Gwenlan, Uta Klein, Johannes Blümlein, Peter Kostka, Frank Marhauser, Oliver Fischer and Nestor Armesto. 


\section{Appendix 1: The Strong Coupling Constant}

There was hardly a talk on deep inelastic scattering where Guido Altarelli would not speak about the importance and difficulty to determine the strong coupling constant. It is indeed amazing to see that we have been working on the determination of $\alpha_{s}$, or $\Lambda_{\overline{M S}}$, since the times of the CDHS and BCDMS and other lepton-hadron scattering experiments, while others worked on $e^{+} e^{-}$or $p p$, that is since 40 years, and yet we are not satisfied with the net result. In Guido's words: The crucial ingredient of perturbative $Q C D$ is the "running" coupling $\alpha_{s}\left(Q^{2}\right) \ldots$ Some particularly simple and clear hard processes must be used to measure the running coupling at a suitable energy scale.. The cleanest processes for measuring $\alpha_{s}$ are the totally inclusive ones (no hadronic corrections) with light cone dominance, like $Z$ decay, scaling violations in DIS and perhaps $\tau$ decay (but, for $\tau$, the energy scale is dangerously low)... In principle DIS is expected to be an ideal laboratory for the determination of $\alpha_{s}$ but in practice the outcome is still to some extent open [76].

\section{Status}

The coupling constant of the strong interaction, $\alpha_{s}$, is much less well known than the fine-structure constant $\alpha$ and the Fermi constant $G_{F}$. Its accurate and more precise determination is a key challenge for experimental and theoretical particle physics, and it is of fundamental importance. This has a number of reasons:

- Approximately, within certain supersymmetric theories, the three couplings approach a point of convergence at the Planck scale. It is the large uncertainty of $\alpha_{s}$ which prevents strong conclusions to be drawn about this unification point as is illustrated in Fig. 11. This sets a goal to reach a ten times higher accuracy with the LHeC, see below.

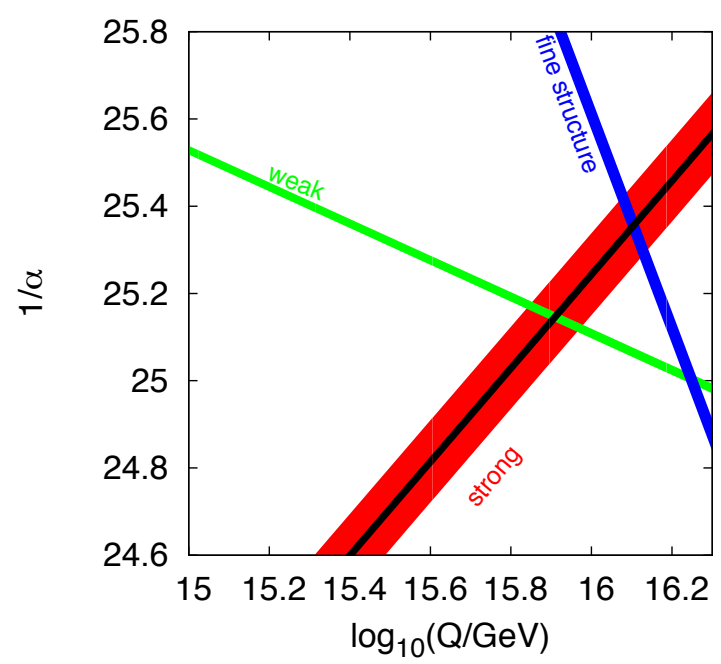

Figure 11: Extrapolation of the inverse coupling constants to the Planck scale, illustrated with a version of MMSM, from [77. The coloured bands represent uncertainties as quoted by PDG in 2012, while the black insert to the red band on $\alpha_{s}$ illustrates the projected LHeC uncertainty, solely from inclusive DIS. The uncertainty of $\alpha_{s}$ quoted by PDG has recently increased by about $50 \%$.

- The current average of various, different determinations of $\alpha_{s}$ is not satisfying which is discussed below and indicated by Guido's remark above. A single, better two independent, consistent measurements of $\alpha_{s}$ at per mille precision are the obvious, though difficult task ahead. In particular, future $e p$ and $e^{+} e^{-}$collider experiments must challenge the lattice results, which also will improve. 
- Deep inelastic scattering has a special role in these determinations. The theory of inclusive DIS is clean, free of hadronic final state phenomenology. In the past it led to peculiarities, due to lack of calibration redundance (BCDMS), smallness of $Q^{2}$ which caused larger power (higher twist) corrections and nuclear and deuteron binding corrections. The most precise DIS data for $\alpha_{s}$, from BCDMS, point to a too low value. For long a tendency had been observed for jet analyses to provide larger values on $\alpha_{s}$ than inclusive ones. All this will be overcome with the LHeC. At that time, DIS theory will have been calculated to $\mathrm{N}^{3} \mathrm{LO}$, and a new level of testing QCD and its development may be near.

- With the LHC reaching high precision in various areas, the uncertainty of $\alpha_{s}$ becomes an obstacle. This holds in particular for the derivation of Higgs couplings at the LHC, because the dominant production process is through gluon-gluon fusion, that is the product of $\alpha_{s} \cdot x g$ enters squared. An uncertainty of $\alpha_{s}\left(M_{Z}^{2}\right)$ taken to be \pm 0.0015 , or $\pm 1.3 \%$, translates to a $2.6 \%$ uncertainty on the cross section 7 as obtained in [78. This assumed uncertainty does not account for the different result obtained by the ABM team, see below. One clearly wishes to determine PDFs and the strong coupling in a process suited to perform a genuine $\mathrm{N}^{3} \mathrm{LO}$ pQCD analysis at a precision which should render effects from $\alpha_{s}$ and the parton distributions, discussed in Sect.5.1, negligible. For Higgs physics at the LHC, this would be achieved with a few per mille accuracy. Similar conclusions one derives when studying the PDF- $\alpha_{s}$ sensitivity of a high precision measurement of the $W$ boson mass [19] or of the weak mixing angle at the LHC.

There exists a vast literature on $\alpha_{s}$ due to its fundamental importance, see e.g. [79, 80, 81]. The current status of the determination of $\alpha_{s}$ has recently been summarised 82 and is reproduced in Tab. 2 using the values as quoted in the PDG2016 [83]. A mean value has been derived of

\begin{tabular}{|c|c|}
\hline Method & $\alpha_{s}\left(M_{Z}^{2}\right)$ \\
\hline Lattice QCD & $0.1184 \pm 0.0012$ \\
$\tau$-decays & $0.1192 \pm 0.0018$ \\
DIS & $0.1156 \pm 0.0021$ \\
Hadron Collider & $0.1151 \pm 0.0028$ \\
Electroweak Fits & $0.1196 \pm 0.0030$ \\
$e^{+} e^{-}$ & $0.1169 \pm 0.0034$ \\
\hline
\end{tabular}

Table 2: Mean values of the strong coupling constant $\alpha_{s}\left(M_{Z}^{2}\right)$, to NNLO and for 5 active flavours 83 .

$\alpha_{s}\left(M_{Z}^{2}\right)=0.1181 \pm 0.0011$ that obviously is dominated by the result of lattice calculations. These have recently been discussed in quite some detail [84]. In the lattice calculations the role of a measured cross section is taken by suitably defined Euclidean short distance quantities. Lattice calculations have a number of additional, common peculiarities, they need input of the experimental hadronic spectrum and quark masses, they treat only light quarks with perturbative, matching additions of charm and beauty quark effects and they have uncertainties from discretization and truncation of perturbative theory. There follows quite a range in the resulting $\alpha_{s}$ values obtained, beyond the simple value of uncertainty quoted, which is achieved by implementing certain quality criteria of the theoretical treatments as are presented in [84]. From a personal point of view one wished to keep the lattice results separate as PDG also does in providing an average of

$$
\alpha_{s}\left(M_{Z}^{2}\right)=0.1174 \pm 0.0016
$$

\footnotetext{
${ }^{7}$ This is consistent with the result presented in [22: using the iHixs code (v1.3) the Higgs prodction cross section in $14 \mathrm{TeV} p p$ Drell-Yan scattering was calculated for NNPDF2.1 using two values of $\alpha_{s}$ which differed by $1.6 \%$. The cross section for 0.121 is larger than the one for 0.119 by about $3.6 \%$. One observes, as expected, that $\delta \sigma / \sigma \simeq 2 \delta \alpha_{s} / \alpha_{s}$.
} 
for all non-lattice results contained in Tab. 2. The next accurate result is that from $\tau$-decays, which, however, as remarked by Guido above, suffers from the small scale, i.e. largeness of $\alpha_{s}(\mu)$ at $\mu=M_{\tau}$ such that fixed order or contour improved perturbation theory lead to $7 \%$ different results, at $M_{\tau}$, and non-perturbative contributions to similarly sizeable uncertainties [83]. The next precise result is from deep inelastic scattering which has a number of partially well known peculiarities the LHeC will eventually resolve.

\section{Deep Inelastic Scattering on $\alpha_{s}$ - Now and with the LHeC}

The status of the determination of $\alpha_{s}$ in DIS is well summarised in Fig.12 taken from a recent comprehensive paper [85] by the ABMP team. A big discrepancy is observed, since long, of the large $\alpha_{s} \simeq 0.121$ value obtained with the SLAC data, at low $Q^{2}$ and large $x$, and the BCDMS result of $\alpha_{s} \simeq 0.11$. The cure to that observation had often been, as in [85] too, the introduction of a higher twist correction for $F_{2}$ which, as a power term $\propto Q^{-4}$, obviously only affects the SLAC result beyond the uncertainty, see Fig. 12 , The fit to all DIS data by ABMP gives an NNLO average of

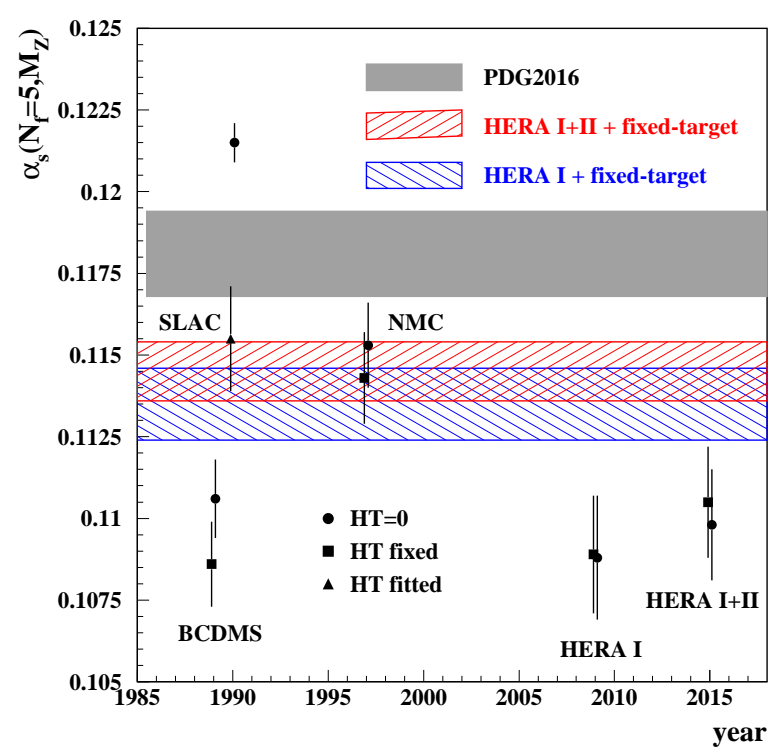

Figure 12: Determinations of $\alpha_{s}$ by the ABMP group [85. One observes quite some spread and a final result (red dashed) below the PDG average value, which includes DIS. Results are shown with the higher twist terms set to zero (circles), HT terms fixed to the values obtained in the ABMP16 fit from considering all data sets (squares) and when fitting the HT terms to the individual data sets (triangles). The bands are obtained by using the combination of the SLAC, BCDMS and NMC samples together with the combined H1 and ZEUS data from HERA I (left-tilted hatches, blue) and from HERA I+II (right-tilted hatches, red).

$$
\alpha_{s}=0.1140 \pm 0.0009
$$

employing HT corrections and for $N_{f}=5$, which is drawn as the red shaded band for the "HERA+fixed target data"-fit. The ABM result thus is (again) lower than the PDG average but consistent with the DIS value quoted from the PDG in Tab. 2. The overall NNLO value of $\alpha_{s}$ the ABMP group obtains, including also hadron collider data, is $0.1147 \pm 0.0008$. This may be compared, as was done in [85], with the MSTW value of $0.1172 \pm 0.0013$ or NNPDF of $0.1174 \pm 0.0007$.

There have been quite some disputes over time about such a low value, the results of which Guido, above, called to some extent still open. It is in my view remarkable that an in depth analysis of the fixed target DIS (SLAC, NMC, BCDMS) and then available data by H1 came to similar 
conclusions as ABMP recently. The H1 Collaboration developed a dedicated procedure [86] for the determination of $\alpha_{s}$ which reduced the number of parton distributions to just two for quarks, owing to the observation that $F_{2}$ in the proton has a singlet, $\Sigma$, and a non-singlet, $\Delta$, component,

$$
F_{2}=\frac{2}{9} \cdot x \Sigma+\frac{1}{3} \cdot x \Delta=\frac{1}{3} \cdot x V+\frac{11}{9} \cdot x A
$$

This introduced two functions $V$ and $A$, which approximately are valence and sea dominated, respectively. One therefore hoped to be able to reduce the uncertainty on $\alpha_{s}$ by reducing the PDF correlations to a minimum. Similar observations to Fig. 12 were made and in much detail described in [87. The cure, however, was chosen to be different: because an unpleasant $Q_{\min }^{2}$ dependence of $\alpha_{s}$ was observed, and to avoid HT terms, a cut of $Q^{2} \geq 7.5 \mathrm{GeV}^{2}$ was implemented for the fixed target data. That was built into the bulk BCDMS data anyhow, as the detector had originally left space for a calorimeter to detect $\mathrm{CC}$ scattering which was never put in place. It was then seen that the NMC data had no further effect on $\alpha_{s}$, due to the more precise BCDMS data. Moreover, inconsistencies were observed and studied at length, see [87], of the slopes in $Q^{2}$ at high $x$ measured by SLAC and BCDMS. This caused the introduction of a cut for the inelasticity $y_{B C D M S}$ to be larger than 0.3. The result was that of full consistency, in the so constrained phase space, such that a $\chi^{2}+1$ criterion was applicable which rarely is the case in global PDF and $\alpha_{s}$ analyses using unpleasant "tolerance criteria" to screen the incompatibilities of data used. In 2001, H1 obtained an NLO $\alpha_{s}$ value of

$$
\alpha_{s}\left(M_{Z}^{2}\right)=0.1150 \pm 0.0017(\exp ) \stackrel{+}{-} 0.00005(\text { model })
$$

which to NNLO pQCD would provide a lower number, probably close to 0.114 as ABMP indeed now obtained. Here, the first error represents the experimental uncertainty on $\alpha_{s}$ of $1.5 \%$. The second, lower error includes all uncertainties associated with the construction of the QCD model for the measured cross section. A number to be noted may be that a $100 \mathrm{MeV}$ uncertainty on the value of the charm mass results in an $\alpha_{s}$ uncertainty of \pm 0.0005 . A program to determine $\alpha_{s}$ to per mille precision thus requires to fix the value of $M_{c}$ to better than $10 \mathrm{MeV}$.

When we on H1 designed our QCD fit strategy, was it in 1990, I recall that the admirable Willy van Neerven advised to keep inclusive and jet data QCD analyses separate. It could be, he argued convincingly, that the gluon determined in inclusive DIS told us something when compared to the jet data result, which obviously concerned $\alpha_{s}$ as well. It was therefore, for very long, avoided to put all together. In 2017, H1 published a comprehensive NNLO analysis of jet and all the inclusive data 88 . It led to the interesting result, for jets only and to NNLO, of

$$
\alpha_{s}\left(M_{Z}^{2}\right)=0.1157 \pm 0.0020(\exp ) \pm 0.0029(\text { thy }) .
$$

A joint fit to jet and inclusive DIS H1 data led to $\alpha_{s}=0.1142 \pm 0.0028$ (tot), in remarkable agreement with ABMP. There again is a tendency to be noted that $\alpha_{s}$ from jets is somewhat higher than from inclusive DIS. This is about as far as HERA will lead and so we studied comprehensively the potential to obtain $\alpha_{s}$ from simulated LHeC data.

Two independent simulations and fit approaches have been undertaken in order to verify the potential of the LHeC to determine $\alpha_{s}$, see the CDR [5] for details. Table 3 summarises the main results. It can be seen that the total experimental uncertainty on $\alpha_{s}$ is $0.2 \%$ from the LHeC and $0.1 \%$ when combined with HERA. This determination is free of higher twist, hadronic and nuclear corrections relying solely on inclusive DIS ep data at high $Q^{2}$. There are known further parametric uncertainties in DIS determinations of $\alpha_{s}$. These can safely be expected to be much reduced also by the LHeC, which promises to determine the charm mass, for example, by $3 \mathrm{MeV}$, as compared to $40 \mathrm{MeV}$ at HERA, corresponding to an $\alpha_{s}$ uncertainty of $0.04 \%$. Matching the experimental uncertainty requires that when the LHeC operates such analyses were performed in $\mathrm{N}^{3} \mathrm{LO}$ pQCD in 


\begin{tabular}{|l|c|c|c|}
\hline case & cut $\left[Q^{2}\left(\mathrm{GeV}^{2}\right)\right]$ & uncertainty & relative precision (\%) \\
\hline HERA only & $Q^{2}>3.5$ & 0.00224 & 1.94 \\
HERA+jets & $Q^{2}>3.5$ & 0.00099 & 0.82 \\
\hline LHeC only & $Q^{2}>3.5$ & 0.00020 & 0.17 \\
LHeC+HERA & $Q^{2}>3.5$ & 0.00013 & 0.11 \\
LHeC+HERA & $Q^{2}>7.0$ & 0.00024 & 0.20 \\
LHeC+HERA & $Q^{2}>10$. & 0.00030 & 0.26 \\
\hline
\end{tabular}

Table 3: Results of NLO QCD fits to HERA data (top, without and with jets) to the simulated LHeC data alone and to their combination, for details of the fit see [5. The resulting uncertainty includes all the statistical and experimental systematic error sources taking their correlations into account. The LHeC result does not include jet data.

order to reduce the renormalisation and factorisation scale uncertainty. Due to the huge range in $Q^{2}$ and the high precision of the data new, decisive tests will also become available for answering the question whether the strong coupling determined with jets and in inclusive DIS are the same. If confirmed, as is demonstrated in Tab.3 with the HERA data, a joint inclusive and jet analysis has the potential to even further reduce the uncertainty of $\alpha_{s}$ derived from future LHeC data. The ambition to measure $\alpha_{s}$ to per mille accuracy therefore represents a vision for a renaissance of the physics of deep inelastic scattering which is a major goal of the whole LHeC enterprise.

\section{Appendix 2: The Longitudinal Structure Function and R}

In 2012, Guido Altarelli publishes an article [89], in honour of Mario Greco, about "The Early Days of QCD (as seen from Rome)" in which he describes one of his main achievements, and long term irritation, regarding the so-called longitudinal structure function, $F_{L}$, and its measurement. Back to Rome I met Guido Martinelli, then a post-doc with a contract for doing accelerator physics at Frascati, and I rescued him into particle physics, with a work on the transverse momentum distributions for jets in lepto-production final states [8]. In the same paper we derived an elegant formula for the longitudinal structure function $F_{L}$, also an effect of order $\alpha_{s}\left(Q^{2}\right)$, as a convolution integral over $F_{2}\left(x, Q^{2}\right)$ and the gluon density $g\left(x, Q^{2}\right)$. I find it surprising that it took 40 years since the start of deep inelastic scattering experiments to get meaningful data on the longitudinal structure function. The present data, recently obtained by the $H 1$ experiment at DESY, are in agreement with this LO QCD prediction but the accuracy of the test is still far from being satisfactory for such a basic quantity.

The measurement of $F_{L}$ concerned me for more than twenty years, from first studies of the sensitivity at HERA in 1988 to the first announcement of results at in Philadelphia ICHEP 2008 [90] and its final publication. Why is $F_{L}$ a quantity of special interest, and why was its determination so difficult at HERA and how would LHeC surpass it?

The inclusive, deep inelastic electron-proton scattering cross section at low $Q^{2}$, written in its reduced form,

$$
\sigma_{r}=\frac{d^{2} \sigma}{d x d Q^{2}} \cdot \frac{Q^{4} x}{2 \pi \alpha^{2} Y_{+}}=F_{2}\left(x, Q^{2}\right)-\frac{y^{2}}{Y_{+}} \cdot F_{L}\left(x, Q^{2}\right)
$$

is defined by two proton structure functions, $F_{2}$ and $F_{L}$, where $Q^{2}$ is the negative four-momentum transfer squared, $y$ the inelasticity $y=Q^{2} / s x \leq 1, Y_{+}=1+(1-y)^{2}$ and $\alpha$ is the fine structure constant. The cross section may also be expressed [91] as a sum of two contributions, $\sigma_{r} \propto\left(\sigma_{T}+\right.$ $\left.\epsilon \sigma_{L}\right)$, referring to the transverse and longitudinal polarisation state of the exchanged boson, with $\epsilon$ characterising the ratio of the longitudinal to the transverse polarisation. The ratio of the longitudinal 
to transverse cross sections is termed

$$
R\left(x, Q^{2}\right)=\frac{\sigma_{L}}{\sigma_{T}}=\frac{F_{L}}{F_{2}-F_{L}}
$$

which is related to $F_{2}$ and $F_{L}$ as given above. Due to the positivity of the cross sections $\sigma_{L, T}$ one observes that $F_{L} \leq F_{2}$. The reduced cross section $\sigma_{r}$, for $Q^{2}$ much below the weak boson masses, is therefore a direct measure of $F_{2}$. That holds apart from a limited region of high $y$ where a contribution of $F_{L}$ may be sizeable. The principal task to measure $F_{L}$ thus requires to precisely measure the inclusive DIS cross section near to $y=1$, and then to disentangle the two structure functions by utilising the $y^{2} / Y_{+}$variation which depends on $x, Q^{2}$ and $s$, that is one may vary $s$ and obtains $F_{2}$ and $F_{L}$ at a common, fixed point of $x, Q^{2}$. That is challenging not only because the $F_{L}$ part is small but because $y=1-E^{\prime} / E_{e}$ is large only for scattered electron energies $E^{\prime}$ much smaller than the electron beam energy $E_{e}$, e.g. $E^{\prime}=2.7 \mathrm{GeV}$ for $y=0.9$ at HERA. In the region where $E^{\prime}$ is a few $\mathrm{GeV}$ only, the electron identification becomes a major problem and the electromagnetic $\left(\pi^{0} \rightarrow \gamma \gamma\right)$ and hadronic background, mainly from unrecognised photo-production, rises enormously.

The interest in a measurement of $F_{L}$, especially at low $x$, is related to the uncertainty in the determination of the gluon distribution, $x g\left(x, Q^{2}\right)$, in pQCD fit extractions of the quark and gluon densities, besides $\alpha_{s}$. On more theoretical grounds, there is an expectation that at small $x$ the linear DGLAP $Q^{2}$ evolution equations, which give $x g$ a certain meaning, may eventually have to be replaced by a BFKL type of law. The gluon density is defined in a theoretical framework which needs to be confirmed or disproven which on the basis of only $F_{2}$ is just not possible. With $F_{2}$ and $F_{L}$, however, one has two principal handles for an extraction of $x g$ and the main motivation for our proposal [92] to operate HERA at lower energies was to test higher order QCD at small $x$.

The theoretically most sound and experimentally most precise determination of the gluon density $x g$ is through the measurement of the $Q^{2}$ dependence of $F_{2}$ in DIS 8 . Their relation is prescribed by the DGLAP equations

$$
\frac{\partial F_{2}\left(x, Q^{2}\right)}{\partial \ln Q^{2}}=\frac{\alpha_{s}\left(Q^{2}\right)}{2 \pi} \int_{x}^{1} d z\left[F_{2}\left(\frac{x}{z}\right) P_{q q}(z)+2 \sum_{i=1}^{N_{f}} e_{i}^{2} \cdot G\left(\frac{x}{z}\right) P_{q G}(z)\right]
$$

with the splitting functions $P, x g=G, e_{i}$ the electric charge of quark $i$, and $N_{f}$ the number of active flavours. At small $x$ the first term is almost negligible. One thus finds that the measurement of the $\ln Q^{2}$ derivative of $F_{2}$ determines $x g$, i.e. one needs very high precision in $F_{2}\left(x, Q^{2}\right)$ combined with a large lever arm in $Q^{2}$ at each value of $x$. The extraction of the $F_{2}$ derivative had been evaluated in quite some detail by the H1 Collaboration when first precise measurements on $F_{2}$ became available, see [86]. It was also instrumental to the discovery [93] of the rise of $x g$ towards low $x$.

The second method to cleanly access $x g$ is through the relation mentioned by Guido

$$
F_{L}\left(x, Q^{2}\right)=\frac{\alpha_{s}}{\pi} x^{2} \int_{x}^{1} \frac{d z}{z^{3}}\left[\frac{4}{3} F_{2}\left(z, Q^{2}\right)+2 \sum_{i}^{N_{f}} e_{i}^{2} \cdot G\left(z, Q^{2}\right)\left(1-\frac{x}{z}\right)\right]
$$

derived in [8], see also [94]. One can calculate that the integrand at small $x$ is dominated by the gluon distribution term which directly relates $F_{L}$ and $x g$.

The final measurement of $F_{L}$ at HERA [95] is illustrated in Fig. 13. The data were obtained in an evolved separation procedure which principally determined $F_{L}$ as the intercept, at $y=0$, and $F_{L}$ as the negative slope of the linear dependence of $\sigma_{r}$ on $y^{2} / Y_{+}$. The variation in $y$ was achieved at HERA by comparing high statistics data at highest energy, $E_{p}=920 \mathrm{GeV}$, with about $13 \mathrm{pb}^{-1}$

\footnotetext{
${ }^{8}$ This is transparent from the sudden increase of uncertainty for $x g$ below $x \simeq 0.0005$, see Fig. 5 . where HERA's access to $\partial F_{2} / \partial \ln Q^{2}$ ends.
} 

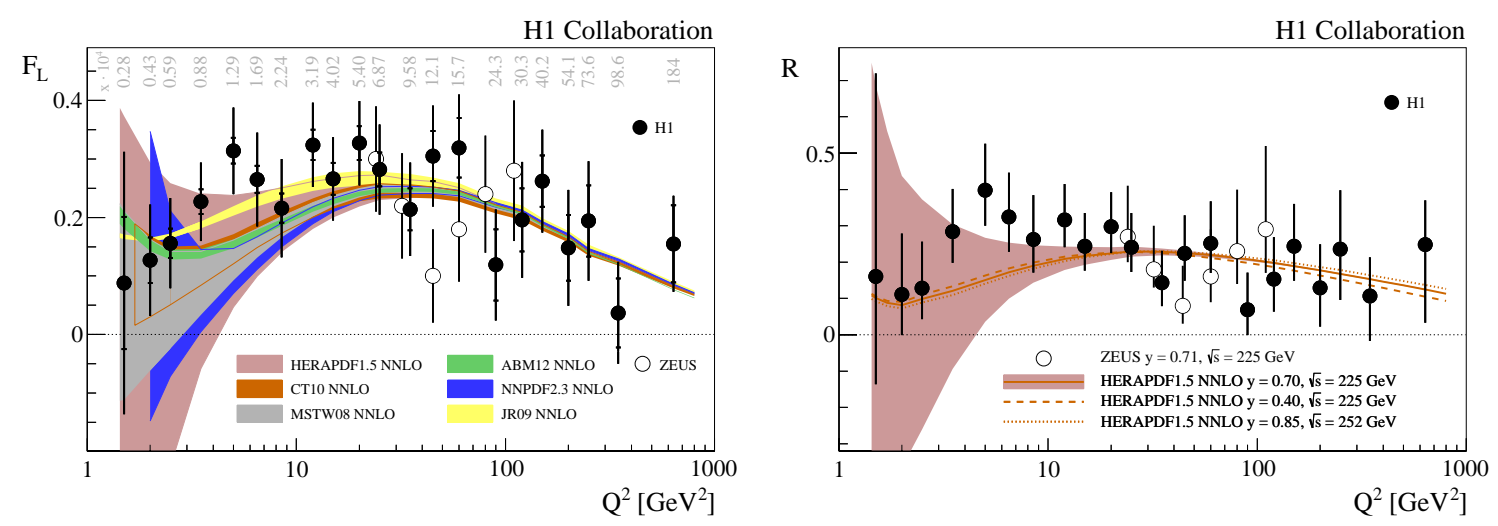

Figure 13: Left: Measurement of the structure function $F_{L}\left(x, Q^{2}\right)$ at H1 (solid points) and ZEUS (open circles), from a variation of proton beam energy in the final half year of HERA operation. The curves and their uncertainty bands illustrate predictions on $F_{L}$ obtained to NNLO pQCD by various groups engaged in the extraction of PDFs. Right: Measurement of the structure function ratio $R=F_{L} /\left(F_{2}-F_{L}\right)$ at H1 (solid points) and ZEUS (open circles), from a variation of proton beam energy in the final half year of HERA operation. The curve represents an NNLO QCD fit analysis of the other HERA data. This becomes uncertain for $Q^{2}$ below $10 \mathrm{GeV}^{2}$ where the $Q^{2}$ dependence of $F_{2}$ does not permit an accurate determination of the gluon density which dominates the prediction on $F_{L}$, see text.

of data at $460 \mathrm{GeV}$ and $7 \mathrm{pb}^{-1}$ at $575 \mathrm{GeV}$. The values of $E_{p}$ had been chosen for about equidistant separation of the measurements in $y^{2} / Y_{+}$. The low energy runs took place from March to June 2007, with only a few days of setup time for the machine. One notices that the absolute, total uncertainty of the $\mathrm{H} 1$ and ZEUS data is about \pm 0.1 , with notable statistical uncertainties for $Q^{2} \geq 20 \mathrm{GeV}^{2}$. The ZEUS data do not extend to low $Q^{2}$ values, which could be accessed by H1 [96] thanks to the existence of the Backward Silicon Tracker which my group had developed [97], built and operated over a time of 15 years. A clear track-cluster link was required to identify low energy scattered electrons near the beam pipe. The determination of the electron's charge, in a demanding, small radius curvature measurement, permitted in addition the removal of a large part of background with a residual account for charge asymmetry effects as are caused by the difference in energy depositions of anti-protons and protons at low energy. As is described in [96 quite refined techniques had to be found and to work for accessing $F_{L}$.

In Fig. 13 the data are compared with predictions, calculated in NNLO pQCD, from various groups who perform comprehensive analyses of parton distribution functions. One observes a generally good agreement and recognises the origin for Guido Altarelli's remark cited above: the data are impressive, however, they are not precise enough for crucial tests of pQCD. In the specially interesting region of low $x$ where the PDF uncertainties, mainly related to $x g$ (see Sect.5.1), are substantial, the data hint to a positive and regular $F_{L}$ but lack discriminative power also. This can also be illustrated well with the measurement of $\sigma_{L} / \sigma_{T}$.

The first measurements of $R$ at SLAC [98, 99], in the range of $Q^{2}$ between 1 and about $10 \mathrm{GeV}^{2}$ and $0.1 \leq x \leq 0.5$, found the ratio to be about 0.18, i.e. small enough to be taken as evidence for quarks to carry spin $1 / 2$. The HERA data determine this ratio as a function of $Q^{2}$, and correspondingly varying $x=Q^{2} /\left(4 E_{e} E_{p} y\right) \simeq Q^{2} /\left(4 \cdot 10^{4} \mathrm{GeV}^{2}\right)$, for $E_{p}=575 \mathrm{GeV}$ and $y \simeq 0.7$. The result is displayed in Fig.13. To good approximation, $R\left(x, Q^{2}\right)$ is a constant which was determined as $R=0.23 \pm 0.04$, in astonishing good agreement with the SLAC values. The resulting value also obeys the condition, $R \leq 0.37$, that had been obtained in a rigorous attempt to derive the dipole model for inelastic DIS [100].

The LHeC will lead to the first precision measurement of $F_{L}$ and $R$. This is due to three main improvements: i) the luminosity is expected to be so large that high statistics, $\mathrm{fb}^{-1}$ measurements can be performed at even more than two low beam energy points, compared with the two $10 \mathrm{pb}^{-1}$ sets at HERA; ii) the apparatus in the backward region is being designed as a high precision device 

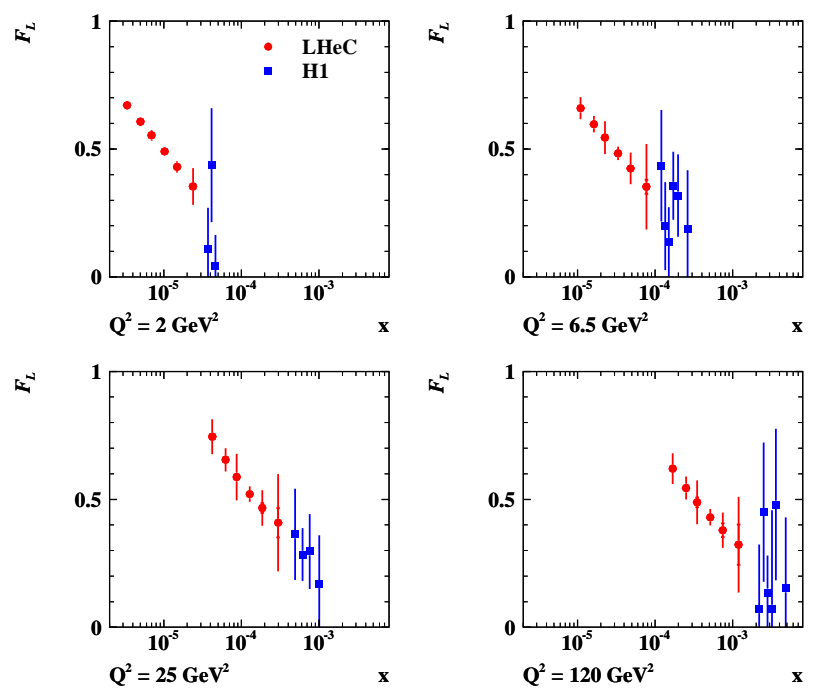

Figure 14: Simulations of $F_{L}$ measurements with the LHeC (red circles) compared with measurements at H1 (blue squares), see text.

with more Silicon detector planes of higher acceptance and resolution and a hadronic backward calorimeter which was basically absent on $\mathrm{H} 1$; iii) the increased electron beam energy implies that high $y$ may be achieved at larger scattered electron energy $E^{\prime}$. Both the improved detector and the enlarged $E_{e}$ will enable to reach highest $y$ values at much reduced background.

A simulation had been performed for the LHeC CDR [5] which is illustrated in Fig. 14. In order to be conceptually independent of the LHC operation, for the LHeC the electron beam energy is lowered as opposed to HERA. The point-by-point precision is impressively improved, from at best $\delta F_{L} \simeq \pm 0.1-0.2$ with $\mathrm{H} 1$ to typically a 0.02 total uncertainty for the LHeC. Based on the invaluable experience gained with $\mathrm{H} 1$ at HERA and on the design prospects for the LHeC and its $e p$ experiment, one can indeed be optimistic that Guido Altarelli's wish for a precise determination of $F_{L}$ will eventually be fulfilled. The simulated data, with their exceptional determinations of $F_{2}$ and $F_{L}$, were used in a study, presented in the CDR, to illustrate the unique potential in discriminating theory at small $x$.

$+$

\section{References}

[1] Elliott D. Bloom et al. High-Energy Inelastic e p Scattering at 6-Degrees and 10-Degrees. Phys. Rev. Lett., 23:930-934, 1969.

[2] Martin Breidenbach, Jerome I. Friedman, Henry W. Kendall, Elliott D. Bloom, D. H. Coward, H. C. DeStaebler, J. Drees, Luke W. Mo, and Richard E. Taylor. Observed Behavior of Highly Inelastic electron-Proton Scattering. Phys. Rev. Lett., 23:935-939, 1969.

[3] Guido Altarelli and G. Parisi. Asymptotic Freedom in Parton Language. Nucl. Phys., B126:298-318, 1977.

[4] Guido Altarelli. The Higgs and the Excessive Success of the Standard Model. Frascati Phys. Ser., 58:102, 2014.

[5] J. Abelleira Fernandez and the LHeC Study Group. A Large Hadron Electron Collider at CERN. Journal of Physics G: Nuclear and Particle Physics, 39(7):075001, 2012.

[6] D. Angal-Kalinin et al. PERLE: Powerful Energy Recovery Linac for Experiments - Conceptual Design Report, J.Phys.G to appear,. arXiv:1705.08783, 2017.

[7] G. Altarelli. Deep Inelastic Scattering in the LHC Time. Opening Lecture, ECFA LHeC Workshop at Divonne, 2008. 
[8] Guido Altarelli and G. Martinelli. Transverse Momentum of Jets in Electroproduction from Quantum Chromodynamics. Phys. Lett., 76B:89-94, 1978.

[9] H. Abramowicz et al. Combination of measurements of inclusive deep inelastic $e^{ \pm} p$ scattering cross sections and QCD analysis of HERA data. Eur. Phys. J., C75(12):580, 2015.

[10] M. Klein and R. Yoshida. Collider Physics at HERA. Prog.Part.Nucl.Phys., 61:343-393, 2008.

[11] M. Klein. The Structure of the Proton and HERA. In Proceedings of PHOTON 2009, May 11-15, 2009 DESY Hamburg, Germany, pages 431-444, 2010.

[12] E. C. Aschenauer, S. Fazio, J. H. Lee, H. Mantysaari, B. S. Page, B. Schenke, T. Ullrich, R. Venugopalan, and P. Zurita. The EIC: Assessing the Energy Dependence of Key Measurements. arXiv:1708.01527, 2017.

[13] S. Abeyratne et al. MEIC Design Summary. arXiv:1504.07961, 2015.

[14] E. C. Aschenauer et al. eRHIC Design Study: An Electron-Ion Collider at BNL. arXiv:1409.1633, 2014.

[15] M. Klein. EIC and LHeC side by side. Invited talk at POETIC, Philadelphia, 2016.

[16] C. Alexandrou, M. Constantinou, K. Hadjiyiannakou, K. Jansen, C. Kallidonis, G. Koutsou, A. Vaquero Avils-Casco, and C. Wiese. The nucleon spin explained using lattice QCD simulations. Phys. Rev. Lett., 119(14):142002, 2017.

[17] G. Aad et al. Expected Performance of the ATLAS Experiment - Detector, Trigger and Physics, 2009.

[18] Morad Aaboud et al. Precision measurement and interpretation of inclusive $W^{+}, W^{-}$and $Z / \gamma^{*}$ production cross sections with the ATLAS detector. Eur. Phys. J., C77(6):367, 2017.

[19] M. Aaboud et al. Measurement of the $W$-boson mass in pp collisions at $\sqrt{s}=7 \mathrm{TeV}$ with the ATLAS detector. arXiv:1701.07240. EPJ C to appear, 2018.

[20] N. Armesto and E. Scomparin. Heavy-ion collisions at the Large Hadron Collider: a review of the results from Run 1. Eur. Phys. J. Plus, 131(3):52, 2016.

[21] Frank Zimmermann, Oliver Bruening, and Max Klein. The LHeC as a Higgs Boson Factory. In Proceedings, 4th International Particle Accelerator Conference (IPAC 2013): Shanghai, China, May 12-17, 2013, page MOPWO054, 2013.

[22] Oliver Bruening and Max Klein. The Large Hadron Electron Collider. Mod. Phys. Lett., A28(16):1330011, 2013.

[23] O. Brüning et al. FCC-eh Baseline Parameters. FCC-ACC-RPT-0012, April 2017.

[24] E. Jensen. Choice of Rf Frequency, Talk at LHeC Workshop, Daresbury. 2016.

[25] F. Marhauser. Choice of Rf Frequency, Talk at PERLE Workshop, Orsay. 2017.

[26] F. Marhauser et al. $802 \mathrm{MHz}$ ERL Cavity Design and Development, Proc. IPAC, to be published. 2018.

[27] Extreme Light Infrastructure. www.eli-np.ro. 2017.

[28] A. Gaddi. Installation of the LHeC Detector, Talk at LHeC Workshop, CERN. 2017.

[29] B. Parker. SC Magnet Concepts for eh Colliders IRs, Talk at LHeC Workshop, CERN. 2017.

[30] John C. Collins, Davison E. Soper, and George F. Sterman. Factorization of Hard Processes in QCD. Adv. Ser. Direct. High Energy Phys., 5:1-91, 1989.

[31] S. Moch, B. Ruijl, T. Ueda, J. A. M. Vermaseren, and A. Vogt. Four-Loop Non-Singlet Splitting Functions in the Planar Limit and Beyond. JHEP, 10:041, 2017.

[32] M. Klein and V. Radescu. Partons from the LHeC. LHeC-Note-2013-002 PHY, Snowmass Workshop, 2013.

[33] Georges Aad et al. Measurement of the inclusive $W^{ \pm}$and $\mathrm{Z} /$ gamma cross sections in the electron and muon decay channels in $p p$ collisions at $\sqrt{s}=7 \mathrm{TeV}$ with the ATLAS detector. Phys. Rev., D85:072004, 2012.

[34] Georges Aad et al. Determination of the strange quark density of the proton from ATLAS measurements of the $W \rightarrow \ell \nu$ and $Z \rightarrow \ell \ell$ cross sections. Phys. Rev. Lett., 109:012001, 2012.

[35] Arthur M. Jaffe and Edward Witten. Quantum Yang-Mills Theory. 2000.

[36] A. Accardi, F. Arleo, W. K. Brooks, David D'Enterria, and V. Muccifora. Parton Propagation and Fragmentation in QCD Matter. Riv. Nuovo Cim., 32:439-553, 2010.

[37] G. Altarelli. Importance of Deep Inelastic Scattering in the 21st Century. Talk presented at the LHeC Workshop, Geneva, CERN, 2015. 
[38] Morad Aaboud et al. Search for a new heavy gauge boson resonance decaying into a lepton and missing transverse momentum in $36 \mathrm{fb}^{-1}$ of $p p$ collisions at $\sqrt{s}=13 \mathrm{TeV}$ with the ATLAS experiment. 2017.

[39] C. Borschensky and M. Kramer. private communication. 2016.

[40] E. Kay and U. Klein. private communication. 2017.

[41] S. Camarda. $M_{W}$ from the LHeC, private communication. 2017.

[42] D. Britzger and M. Klein. Electroweak Physics with Inclusive DIS Data at the LHeC and FCC-eh, Proceedings DIS2017, to appear. 2017.

[43] Guido Altarelli. The Higgs: so simple yet so unnatural. EPJ Web Conf., 71:00005, 2014.

[44] ATLAS Collaboration. ATL-PHYS-PUB-2014-016. 2014.

[45] M. Klein and U. Klein. SM Higgs Couplings in ep, in preparation. 2018.

[46] Albert M Sirunyan et al. Evidence for the Higgs boson decay to a bottom quark-antiquark pair. arXiv:1709.07497, 2017.

[47] M. Aaboud et al. Evidence for the $H \rightarrow b \bar{b}$ decay with the ATLAS detector. JHEP, 12:024, 2017.

[48] U. Klein. Turning the LHC into a Powerful Higgs Facility, Seminar at LAPP Annecy. http : //lhec.web.cern.ch. 2017.

[49] U. Klein. Talk on Higgs Physics at the LHeC Workshop at CERN. 2017.

[50] Guenter Grindhammer, D. Haidt, J. Ohnemus, J. Vermaseren, and D. Zeppenfeld. Searching for the Higgs in e p collisions at LEP / LHC. In ECFA Large Hadron Collider Workshop, Aachen, Germany, 4-9 Oct 1990: Proceedings.2., pages 967-985, 1990.

[51] J. Blumlein, G. J. van Oldenborgh, and R. Ruckl. QCD and QED corrections to Higgs boson production in charged current e p scattering. Nucl. Phys., B395:35-59, 1993.

[52] Tim Barklow, Keisuke Fujii, Sunghoon Jung, Robert Karl, Jenny List, Tomohisa Ogawa, Michael E. Peskin, and Junping Tian. Improved Formalism for Precision Higgs Coupling Fits. 2017.

[53] Stefan Antusch, Eros Cazzato, and Oliver Fischer. Sterile neutrino searches at future $e^{-} e^{+}, p p$, and $e^{-} p$ colliders. Int. J. Mod. Phys., A32(14):1750078, 2017.

[54] Mukesh Kumar, Xifeng Ruan, Rashidul Islam, Alan S. Cornell, Max Klein, Uta Klein, and Bruce Mellado. Probing anomalous couplings using di-Higgs production in electronproton collisions. Phys. Lett., B764:247-253, 2017.

[55] David Curtin, Kaustubh Deshpande, Oliver Fischer, and Jose Zurita. New Physics Opportunities for Long-Lived Particles at Electron-Proton Colliders. arXiv:1712.07135, 2017.

[56] Shang Liu, Yi-Lei Tang, Chen Zhang, and Shou-hua Zhu. Exotic Higgs Decay $h \rightarrow \phi \phi \rightarrow 4 b$ at the LHeC. Eur. Phys. J., C77(7):457, 2017.

[57] Baradhwaj Coleppa, Mukesh Kumar, Satendra Kumar, and Bruce Mellado. Measuring CP nature of top-Higgs couplings at the future Large Hadron electron collider. Phys. Lett., B770:335-341, 2017.

[58] Georges Azuelos, Hao Sun, and Kechen Wang. Search for Singly Charged Higgs in Vector Boson Scattering at the ep Colliders. arXiv:1712.07505, 2017.

[59] M. Trott. Physics of the Higgs Boson, Talk at the LHeC Workshop at Chavannes-de-Bogis. 2014.

[60] O. Cakir. Anomalous Triple Gauge Couplings, Talk at the FCC Physics Workshop at CERN. 2017.

[61] Hao Sun and XiaoJuan Wang. Searches for the Anomalous FCNC Top-Higgs Couplings at the LHeC. 2016.

[62] Hao Sun. Top Physics at the LHeC, Talk at the DIS Workshop, Birmingham. 2017.

[63] C. Schwanenberger. Top Physics at the LHeC, Talk at the LHeC Workshop at CERN. 2017.

[64] S. Forte. LHeC: The Ultimate Factorization Machine, Talk at the LHeC Workshop at Chavannes-de-Bogis. 2012.

[65] C. Quigg. DIS and Beyond, Summary Talk at the DIS Workshop at Marseille. 2013.

[66] Guido Altarelli, Richard D. Ball, and Stefano Forte. Small x Resummation with Quarks: Deep-Inelastic Scattering. Nucl. Phys., B799:199-240, 2008.

[67] L. V. Gribov, E. M. Levin, and M. G. Ryskin. Semihard Processes in QCD. Phys. Rept., 100:1-150, 1983. 
[68] M. Strikman. Probing BFKL Dynamics in eh Scattering in large t semi-inclusive Rapidity Gap Processes, Talk at the LHeC Workshop at Chavannes-de-Bogis. 2015.

[69] A. Accardi et al. Electron Ion Collider: The Next QCD Frontier. Eur. Phys. J., A52(9):268, 2016.

[70] Stanley J. Brodsky. Novel features of nuclear chromodynamics. Eur. Phys. J., A53(3):48, 2017.

[71] N. Armesto. Electron-Ion Physics: Status Report, Talk at the LHeC Workshop at CERN. 2017.

[72] Max Klein. Nuclear Parton Distributions with the LHeC. EPJ Web Conf., 112:03002, 2016.

[73] Ilkka Helenius, Hannu Paukkunen, and Nestor Armesto. nPDF constraints from the Large Hadron Electron Collider. PoS, DIS2016:276, 2016.

[74] N. Armesto. Private Communication.

[75] G. Altarelli. Remark at the Chavannes-de-Bogis Session of the LHeC Advisory Committee. 2015.

[76] Guido Altarelli. The QCD Running Coupling and its Measurement. PoS, Corfu2012:002, 2013.

[77] J. L. Abelleira Fernandez et al. On the Relation of the LHeC and the LHC. arXiv:1211.5102, 2012.

[78] Charalampos Anastasiou, Claude Duhr, Falko Dulat, Elisabetta Furlan, Thomas Gehrmann, Franz Herzog, Achilleas Lazopoulos, and Bernhard Mistlberger. High precision determination of the gluon fusion Higgs boson cross-section at the LHC. JHEP, 05:058, 2016.

[79] Alexandre Deur, Stanley J. Brodsky, and Guy F. de Teramond. The QCD Running Coupling. Prog. Part. Nucl. Phys., 90:1-74, 2016.

[80] G. Dissertori. The Determination of the Strong Coupling Constant. Adv. Ser. Direct. High Energy Phys., 26:113-128, 2016.

[81] David d'Enterria, editor. Proceedings, High-Precision $\alpha_{s}$ Measurements from LHC to FCC-ee, Geneva, 2015. CERN, CERN.

[82] S. Bethke. $\alpha_{s}$ 2016. Nucl. Part. Phys. Proc., 282-284:149-152, 2017.

[83] C. Patrignani et al. Review of Particle Physics. Chin. Phys., C40(10):100001, 2016.

[84] S. Aoki et al. Review of lattice results concerning low-energy particle physics. Eur. Phys. J., C77(2):112, 2017.

[85] S. Alekhin, J. Blmlein, S. Moch, and R. Placakyte. Parton distribution functions, $\alpha_{s}$, and heavy-quark masses for LHC Run II. Phys. Rev., D96(1):014011, 2017.

[86] C. Adloff et al. Deep inelastic inclusive e p scattering at low x and a determination of alpha(s). Eur. Phys. J., C21:33-61, 2001.

[87] Rainer Wallny. A Measurement of the Gluon Distribution in the Proton and of the Strong Coupling Constant $\alpha_{s}$ from Inclusive Deep-Inelastic Scattering. PhD thesis, Zurich U., 2001.

[88] V. Andreev et al. Determination of the strong coupling constant $\alpha_{s}\left(m_{Z}\right)$ in next-to-next-to-leading order QCD using H1 jet cross section measurements. Eur. Phys. J., C77(11):791, 2017.

[89] G. Altarelli. The Early Days of QCD (as seen from Rome). Nuovo Cim., C035N1:1-8, 2012.

[90] Max Klein. First Measurements of F(L) at Low Bjorken x. In Proceedings, 34th International Conference on High Energy Physics (ICHEP 2008): Philadelphia, Pennsylvania, July 30-August 5, 2008, 2008.

[91] L. N. Hand, D. G. Miller, and Richard Wilson. Electric and Magnetic Formfactor of the Nucleon. Rev. Mod. Phys., 35:335, 1963.

[92] H1 Collaboration. Running at Low Proton Beam Energies. H1-10/05-622, 2005.

[93] I. Abt et al. Scaling violations of the proton structure function f2 at small x. Phys. Lett., B321:161-167, 1994.

[94] M. Gluck, E. Hoffmann, and E. Reya. Scaling Violations and the Gluon Distribution of the Nucleon. Z. Phys., C13:119, 1982.

[95] V. Andreev et al. Measurement of inclusive ep cross sections at high $Q^{2}$ at $\sqrt{s}=225$ and $252 \mathrm{GeV}$ and of the longitudinal proton structure function $F_{L}$ at HERA. Eur. Phys. J., C74(4):2814, 2014.

[96] F. D. Aaron et al. Measurement of the Inclusive e+-p Scattering Cross Section at High Inelasticity y and of the Structure Function FL. Eur. Phys. J., C71:1579, 2011.

[97] W. Eick et al. Development of the H1 backward silicon strip detector. Nucl. Instrum. Meth., A386:81-86, 1997.

[98] Guthrie Miller et al. Inelastic electron-Proton Scattering at Large Momentum Transfers. Phys. Rev., D5:528, 1972. 
[99] E. M. Riordan, A. Bodek, Martin Breidenbach, D. L. Dubin, J. E. Elias, Jerome I. Friedman, Henry W. Kendall, J. S. Poucher, M. R. Sogard, and D. H. Coward. Extraction of $r=\operatorname{Sigma}(L) / \operatorname{Sigma}(T)$ from Deep Inelastic e p and e d Cross-Sections. Phys. Rev. Lett., 33:561, 1974.

[100] Carlo Ewerz and Otto Nachtmann. Towards a nonperturbative foundation of the dipole picture. II. High energy limit. Annals Phys., 322:1670-1726, 2007. 